\title{
ARL ACADEMIC HeAlth SCIENCES LiBRARY STATISTICS 2008-2009
}

\author{
Compiled and Edited by \\ Martha Kyrillidou \\ SHANEKA MorRIS
}

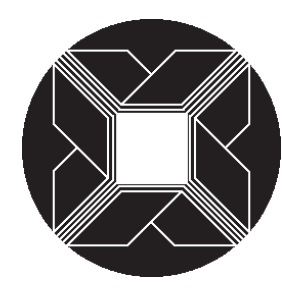

Association of RESEARCH LIBRARIES

WASHINGTON, DC 2011 
ARL Academic Health Sciences Library Statistics 2008-2009

The tables presented in this publication are not indicative of performance and outcomes and should not be used as measures of library quality. In comparing any individual library to ARL medians or to other ARL members, one must be careful to make such comparisons within the context of differing institutional and local goals and characteristics.

The ARL Academic Health Sciences Library Statistics datafiles and accompanying documentation are available at www.arl.org/stats/annualsurveys/med/index.shtml.

Published by the Association of Research Libraries

Washington, DC 20036

www.arl.org

ISSN 1538-9006

ISBN 1-59407-861-0

EAN 978-159407-861-3

\section{(C) 2011}

The compilation is copyrighted by the Association of Research Libraries. Blanket permission is granted to reproduce and distribute copies of this work for nonprofit, educational, or library purposes, provided that the author, source, and copyright notice are included on each copy. This permission is in addition to rights of reproduction granted under Sections 107, 108, and other provisions of the US Copyright Act.

The paper used in this publication meets the minimum requirements of the American National Standard for Information Science and National Information Standards Organization standard-Permanence of Paper for Publications and Documents in Libraries and Archives, ANSI/NISO Z39.48-1992(R1997). 


\section{Contents}

Highlights: ARL Academic Health Sciences Library Statistics 2008-2009 .................................... 5

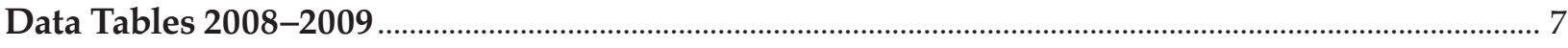

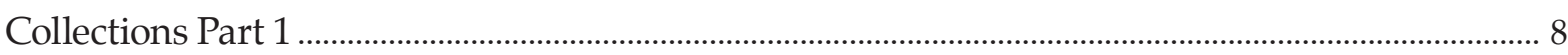

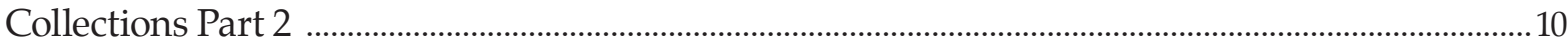

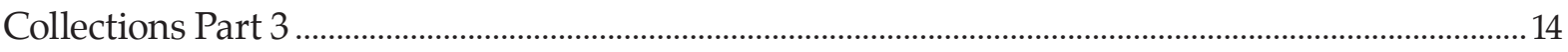

Expenditure

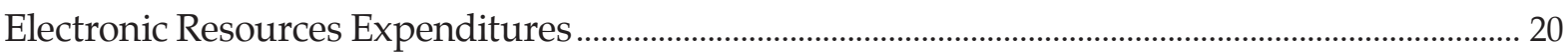

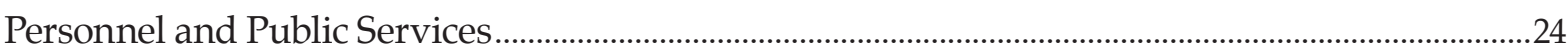

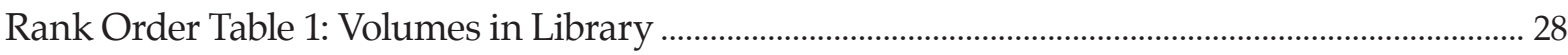

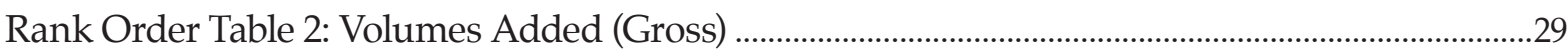

Rank Order Table 3: Current Serials (Total) ……........................................................................................ 30

Rank Order Table 4: Total Library Expenditures........................................................................................31

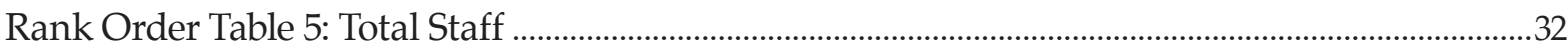

Rank Order Table 6: Total Electronic Resources Expenditures............................................................ 33

ARL Statistics Questionnaire 2008-2009................................................................................................. 35

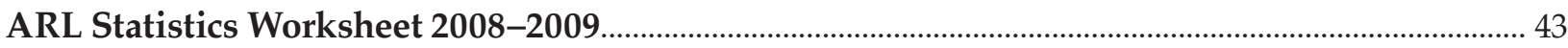

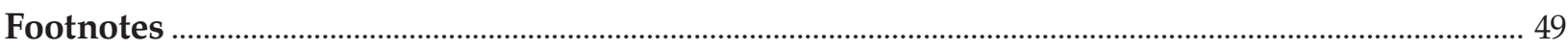

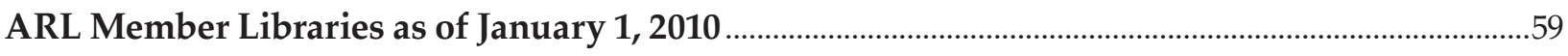





\section{Highlights}

\section{ARL Academic Health Sciences Library Statistics 2008-2009}

- Out of 114 ARL university libraries, 62 responded to this survey. ${ }^{1}$

- Health Sciences libraries reported median values of 230,011 volumes held and 2,249 gross volumes added. Also, these libraries employed the full-time equivalent of 2,131 staff members in the fiscal year 2008-2009.

- Responding libraries reported total expenditures of $\$ 235,821,026 .^{2}$ As seen in the graph below, materials expenditures made up the largest portion of the total, with approximately $50 \%$ of aggregated expenses falling under a materials-related category.

- Health sciences libraries reported a total of $\$ 83,986,222$ in electronic materials expenditures, or a median of almost $79 \%$ of their total materials budgets. This includes a total of $\$ 78,539,253$ in electronic serials expenditures.

\section{Expenditures in ARL Academic Health Sciences Libraries 2008-2009}

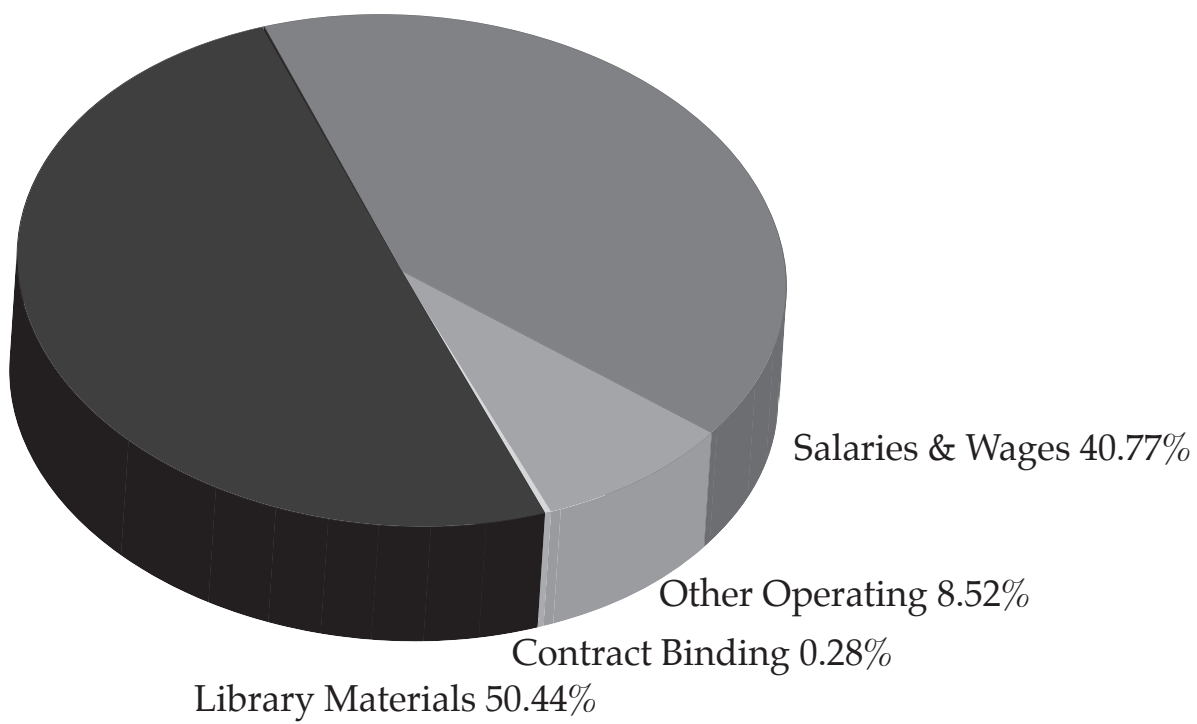

1 Seventy-four ARL university libraries included data for a health sciences library in the 2008-2009 ARL Statistics. Among them, Alberta, British Columbia, Brown, Calgary, Johns Hopkins, Laval, Louisville, Manitoba, Missouri, Ohio, Pittsburgh, SUNY-Buffalo, Toronto, and Wayne State did not complete this survey. Pittsburgh and SUNY-Buffalo completed this survey last year, but chose not to complete this survey in 2008-2009. Cornell and Indiana completed this survey but did not include health sciences library data in the ARL Statistics.

2 This figure includes Canadian universities, whose expenditures were converted to US dollars at the rate of 1.1667 Can $\$=1$ US\$, the average monthly noon exchange rate published in the Bank of Canada Review for the period July 2008 to June 2009. 

Data Tables

2008-2009 


\section{Collections Part 1}

Volumes In Volumes Added Volumes Added Library

(1)
(Net)
Titles Held Monographs Purchased

(Volumes)
(1b)
(1b.i)
(2)

(3)

\section{INSTITUTION}

\begin{tabular}{|c|c|c|c|c|c|c|}
\hline ALABAMA & bG+ & 21,322 & 295 & 247 & . & 233 \\
\hline ARIZONA & bG+ & 222,477 & 1,090 & $-3,701$ & 95,171 & 116 \\
\hline BOSTON & & 145,148 & 1,952 & -51 & 27,772 & 225 \\
\hline CALIFORNIA, DAVIS & $\mathrm{b}+$ & 374,218 & 5,028 & 3,382 & 120,568 & $\mathrm{UA} / \mathrm{NA}$ \\
\hline CALIFORNIA, IRVINE & bG & 377,512 & 11,908 & 11,547 & 252,896 & 4,251 \\
\hline CALIFORNIA, LOS ANGELES & bG+ & 670,751 & 5,532 & 5,363 & 670,751 & 1,149 \\
\hline CALIFORNIA, SAN DIEGO & bG+ + & 229,450 & 3,832 & $-11,258$ & $\mathrm{UA} / \mathrm{NA}$ & $\mathrm{UA} / \mathrm{NA}$ \\
\hline CASE WESTERN RESERVE & G+ & 423,707 & 2,024 & 2,024 & 118,483 & 945 \\
\hline CHICAGO & G+ & 854,643 & 18,830 & 16,114 & 390,940 & 1,859 \\
\hline CINCINNATI & $\mathrm{b}+$ & 293,192 & 1,769 & $-9,648$ & 55,545 & 723 \\
\hline COLUMBIA & + & 338,436 & 1,995 & $-2,631$ & 144,287 & 1,824 \\
\hline CONNECTICUT & G+ & 198,485 & 2,795 & 1,310 & 61,497 & 1,345 \\
\hline CORNELL & + & 201,919 & 2,297 & 1,618 & 63,144 & $\mathrm{UA} / \mathrm{NA}$ \\
\hline DARTMOUTH & bG+ & 260,542 & 1,887 & -395 & 78,528 & 1,103 \\
\hline DUKE & $\mathrm{b}$ & 247,664 & 1,955 & $-52,641$ & . & 357 \\
\hline EMORY & + & 241,736 & 2,560 & 1,408 & 68,212 & 1,072 \\
\hline FLORIDA & + & 319,367 & 1,459 & $-32,855$ & $\mathrm{UA} / \mathrm{NA}$ & 353 \\
\hline FLORIDA STATE & $\mathrm{b}+$ & 12,344 & 3,648 & 3,648 & 5,002 & 116 \\
\hline GEORGE WASHINGTON & bG+ & 98,900 & 1,086 & $-31,599$ & 28,565 & 1,098 \\
\hline GEORGETOWN & $\mathrm{b}+$ & 98,598 & 180 & $-1,374$ & 98,598 & 100 \\
\hline HARVARD & & 695,415 & 8,970 & 8,620 & . & \\
\hline HAWAII & $\mathrm{b}+$ & 33,766 & 1,468 & 1,451 & 9,961 & 5,843 \\
\hline HOWARD & bG+ & 135,021 & 1,145 & 1,013 & 136,718 & 1,186 \\
\hline ILLINOIS, CHICAGO & + & 748,567 & 15,118 & 7,092 & 318,834 & 5,237 \\
\hline INDIANA & bG+ + & 207,693 & 842 & $-80,044$ & 168,798 & 13,675 \\
\hline IOWA & $\mathrm{b}+$ & 359,484 & 11,421 & 8,926 & UA/NA & 498 \\
\hline KANSAS & + & 175,370 & 1,850 & -688 & 61,011 & 463 \\
\hline KENTUCKY & G+ & 243,486 & 2,129 & 1,905 & 97,564 & 1,412 \\
\hline LOUISIANA STATE & + & 49,090 & 315 & 282 & . & 94 \\
\hline MCGILL & $\mathrm{bG}+$ & 485,277 & 13,595 & $-10,480$ & 246,345 & 7,902 \\
\hline MCMASTER & $\mathrm{b}+$ & 144,737 & 1,375 & -178 & 144,737 & $\mathrm{UA} / \mathrm{NA}$ \\
\hline MIAMI & $\mathrm{G}+$ & 207,845 & 1,353 & $-9,836$ & 69,335 & 591 \\
\hline MICHIGAN & bG + & 633,442 & 94,764 & 76,946 & 397,642 & 28,913 \\
\hline MINNESOTA & $\mathrm{b}+$ & 551,030 & 5,641 & 2,960 & $\mathrm{UA} / \mathrm{NA}$ & 1,782 \\
\hline MONTREAL & G+ & 335,595 & 4,028 & $-2,046$ & 151,996 & 2,889 \\
\hline NEW MEXICO & + & 154,661 & 1,099 & 411 & 47,462 & 1,099 \\
\hline NEW YORK & $\mathrm{bG}+$ & 230,572 & 7,820 & 6,658 & 77,710 & 704 \\
\hline NORTH CAROLINA & G & 341,582 & 2,778 & 2,553 & 133,792 & 1,372 \\
\hline NORTHWESTERN & G+ & 292,465 & 3,332 & 3,038 & 145,731 & 1,391 \\
\hline OHIO STATE & $\mathrm{bG}$ & 288,002 & 4,419 & 4,009 & 75,784 & 2,627 \\
\hline OKLAHOMA & + & 329,056 & 5,939 & 5,658 & 102,371 & 2,200 \\
\hline OKLAHOMA STATE & + & 60,392 & 951 & 951 & 90,588 & 960 \\
\hline
\end{tabular}

8- ARL Academic Health Sciences Library Statistics 2008-2009 16 25

251 1,149

33 


\section{Collections Part 1}

1,477

4,002

2,298

142,409

270,949

bG+

$+\quad 191,013$

SASKATCHEWAN

SOUTH CAROLINA

SOUTHERN CALIFORNIA

SOUTHERN ILLINOIS

SUNY-STONY BROOK

TEMPLE

TENNESSEE

TEXAS TECH

TULANE

UTAH

VANDERBILT

VIRGINIA

WASHINGTON

WASHINGTON U.-ST. LOUIS

WISCONSIN

YALE

ALE

MeAn

MEDIAN

$\mathrm{HiGH}$

Low

TOTALS

Number of Libraries Reporting

+- See footnotes

UA/NA - Unavailable or Not Applicable

Notes

\section{0,829}

230,011

854,643

12,344

$16,791,375$

62
Volumes In Volumes Added Volumes Added Library

(1)

(Gross)

(1b.i)

\begin{tabular}{|c|c|c|c|c|c|}
\hline G & 204,395 & 1,477 & 808 & $\mathrm{UA} / \mathrm{NA}$ & UA/NA \\
\hline $\mathrm{b}+$ & 48,493 & 4,002 & $-63,415$ & 23,741 & 345 \\
\hline+ & 142,409 & 2,298 & 408 & UA/NA & 1,077 \\
\hline bG+ & 270,949 & 2,018 & 1,841 & UA/NA & 618 \\
\hline \multirow[t]{2}{*}{+} & 191,013 & 2,693 & 1,716 & 51,368 & 1,725 \\
\hline & 117,118 & 861 & 641 & 40,814 & 186 \\
\hline \multirow[t]{2}{*}{ G+ } & 224,275 & 3,066 & 2,831 & 68,090 & 1,521 \\
\hline & 168,406 & 2,924 & $-3,488$ & 63,343 & 1,286 \\
\hline$b+$ & 274,334 & 708 & 708 & 708 & 648 \\
\hline$b+$ & 145,124 & 2,201 & 1,555 & 56,765 & 2,369 \\
\hline bG+ & 221,223 & 2,183 & 500 & 52,333 & 272 \\
\hline$b+$ & 314,234 & 5,019 & 4,309 & UA/NA & 2,659 \\
\hline \multirow{3}{*}{ bG } & 158,988 & 640 & 640 & & 640 \\
\hline & 197,641 & 1,423 & $-1,602$ & 72,661 & 377 \\
\hline & 195,901 & 698 & $-8,300$ & 73,391 & 200 \\
\hline+ & 124,925 & 2,654 & -426 & $\mathrm{UA} / \mathrm{NA}$ & 1,893 \\
\hline+ & 353,626 & 15,309 & 14,590 & $\mathrm{UA} / \mathrm{NA}$ & 756 \\
\hline bG+ & 298,938 & 3,195 & -598 & 298,938 & 1,870 \\
\hline bG+ & 318,124 & 1,716 & 892 & UA/NA & 508 \\
\hline G+ & 488,300 & 26,087 & 26,062 & 303,869 & 3,455 \\
\hline
\end{tabular}

\section{Summary Data}

\section{5,543}

2,249

94,764

180

343,646

62
(Net)

(1b)
Titles Held Monographs Purchased

(Volumes)

(3)

(2) 


\section{Collections Part 2}

\section{Current Serials}

Total

(Survey Question Number)

Notes

\begin{tabular}{|c|c|c|c|c|c|}
\hline INSTITUTION & Notes & & & & \\
\hline ALABAMA & $\mathrm{bG}+$ & 2,816 & 222 & 97 & 125 \\
\hline ARIZONA & $\mathrm{bG}+$ & 10,785 & 8,680 & 8,383 & 297 \\
\hline BOSTON & & 6,508 & 6,283 & 4,242 & 2,041 \\
\hline CALIFORNIA, DAVIS & $\mathrm{b}+$ & 1,343 & 1,078 & UA/NA & 1,078 \\
\hline CALIFORNIA, IRVINE & bG & 6,466 & 4,315 & 3,882 & 433 \\
\hline CALIFORNIA, LOS ANGELES & bG+ & UA/NA & $\mathrm{UA} / \mathrm{NA}$ & UA/NA & $\mathrm{UA} / \mathrm{NA}$ \\
\hline CALIFORNIA, SAN DIEGO & bG+ & UA/NA & UA/NA & UA/NA & UA/NA \\
\hline CASE WESTERN RESERVE & G+ & 91 & 91 & 0 & 91 \\
\hline CHICAGO & G+ & 29,290 & 26,790 & 25,450 & 1,340 \\
\hline CINCINNATI & $\mathrm{b}+$ & UA/NA & UA/NA & $\mathrm{UA} / \mathrm{NA}$ & UA/NA \\
\hline COLUMBIA & + & UA/NA & UA/NA & UA/NA & UA/NA \\
\hline CONNECTICUT & G+ & 16,586 & 1,765 & 1,158 & 607 \\
\hline CORNELL & + & 9,500 & $\mathrm{UA} / \mathrm{NA}$ & UA/NA & UA/NA \\
\hline DARTMOUTH & bG+ & 3,136 & 1,696 & 1,485 & 211 \\
\hline DUKE & $\mathrm{b}$ & 5,108 & 1,015 & 928 & 87 \\
\hline EMORY & + & 9,087 & 2,038 & 1,895 & 143 \\
\hline FLORIDA & + & 211 & 201 & $\mathrm{UA} / \mathrm{NA}$ & 201 \\
\hline FLORIDA STATE & $b+$ & 698 & 698 & 689 & 9 \\
\hline GEORGE WASHINGTON & bG+ & 2,136 & 963 & 923 & 40 \\
\hline GEORGETOWN & $\mathrm{b}+$ & 4,153 & 3,145 & 3,119 & 26 \\
\hline HARVARD & & $\mathrm{UA} / \mathrm{NA}$ & $\mathrm{UA} / \mathrm{NA}$ & UA/NA & UA/NA \\
\hline HAWAII & $\mathrm{b}+$ & 4,688 & 1,327 & 1,253 & 74 \\
\hline HOWARD & bG & 6,688 & 1,182 & 612 & 570 \\
\hline ILLINOIS, CHICAGO & + & 1,238 & 1,218 & UA/NA & 1,218 \\
\hline INDIANA & bG+ & 7,105 & 1,723 & 1,698 & 25 \\
\hline IOWA & $\mathrm{b}+$ & 5,788 & 3,407 & 3,267 & 140 \\
\hline KANSAS & + & 920 & 883 & 759 & 124 \\
\hline KENTUCKY & G+ & 222 & 212 & UA/NA & 212 \\
\hline LOUISIANA STATE & + & 3,212 & 3,202 & 3,172 & 30 \\
\hline MCGILL & bG+ & 6,792 & 6,089 & 5,555 & 534 \\
\hline MCMASTER & $\mathrm{b}+$ & 293 & 276 & UA/NA & 276 \\
\hline MIAMI & $\mathrm{G}^{+}$ & 9,910 & 9,829 & 9,740 & 89 \\
\hline MICHIGAN & bG+ & 30,971 & 30,589 & 29,880 & 709 \\
\hline MINNESOTA & $\mathrm{b}+$ & $\mathrm{UA} / \mathrm{NA}$ & $\mathrm{UA} / \mathrm{NA}$ & UA/NA & UA/NA \\
\hline MONTREAL & $\mathrm{G}^{+}$ & 3,836 & 3,790 & 3,415 & 375 \\
\hline NEW MEXICO & + & 2,039 & 1,991 & 1,916 & 75 \\
\hline NEW YORK & $\mathrm{bG}+$ & UA/NA & UA/NA & UA/NA & UA/NA \\
\hline NORTH CAROLINA & G & 4,096 & 3,029 & 2,608 & 421 \\
\hline NORTHWESTERN & G+ & 9,644 & 9,644 & UA/NA & UA/NA \\
\hline OHIO STATE & bG & 6,411 & 3,151 & 2,924 & 227 \\
\hline OKLAHOMA & + & UA/NA & UA/NA & UA/NA & UA/NA \\
\hline OKLAHOMA STATE & + & UA/NA & UA/NA & UA/NA & $\mathrm{UA} / \mathrm{NA}$ \\
\hline
\end{tabular}

10 - ARL Academic Health Sciences Library Statistics 2008-2009
Current Serials

Purchased

(5)
Current Serials

Purchased

Electronic

(5a.i)
Current Serials

Purchased Print

(5a.ii) 


\section{Collections Part 2}

Current Serials

Not Purchased

(5b)
Current Serials

Not Purchased Consortial
Current Serials

Not Purchased

Freely Accessible
Current Serials

Not Purchased

Print (5b.ii)
Current Serials

Not Purchased GovDocs

\begin{tabular}{|c|c|c|c|c|c|}
\hline 2,594 & 14 & 2,580 & 0 & 0 & ALABAMA \\
\hline 2,105 & 0 & 2,095 & 10 & 0 & ARIZONA \\
\hline 225 & 0 & 225 & 0 & 0 & BOSTON \\
\hline 265 & $\mathrm{UA} / \mathrm{NA}$ & 265 & UA/NA & $\mathrm{UA} / \mathrm{NA}$ & CALIFORNIA, DAVIS \\
\hline 2,151 & $\mathrm{UA} / \mathrm{NA}$ & 457 & 10 & 1,684 & CALIFORNIA, IRVINE \\
\hline $\mathrm{UA} / \mathrm{NA}$ & $\mathrm{UA} / \mathrm{NA}$ & $\mathrm{UA} / \mathrm{NA}$ & UA/NA & UA/NA & CALIFORNIA, LOS ANGELES \\
\hline $\mathrm{UA} / \mathrm{NA}$ & UA/NA & UA/NA & UA/NA & UA/NA & CALIFORNIA, SAN DIEGO \\
\hline 0 & 0 & 0 & 0 & 0 & CASE WESTERN RESERVE \\
\hline 2,500 & 0 & 2,500 & 0 & UA/NA & CHICAGO \\
\hline $\mathrm{UA} / \mathrm{NA}$ & UA/NA & UA/NA & UA/NA & UA/NA & CINCINNATI \\
\hline $\mathrm{UA} / \mathrm{NA}$ & $\mathrm{UA} / \mathrm{NA}$ & $\mathrm{UA} / \mathrm{NA}$ & UA/NA & UA/NA & COLUMBIA \\
\hline 14,821 & 14,321 & 500 & 0 & 0 & CONNECTICUT \\
\hline $\mathrm{UA} / \mathrm{NA}$ & UA/NA & $\mathrm{UA} / \mathrm{NA}$ & UA/NA & UA/NA & CORNELL \\
\hline 1,440 & UA/NA & 1,259 & 85 & 96 & DARTMOUTH \\
\hline 4,093 & 3,281 & 808 & 4 & 0 & DUKE \\
\hline 7,049 & $\mathrm{UA} / \mathrm{NA}$ & $\mathrm{UA} / \mathrm{NA}$ & $\mathrm{UA} / \mathrm{NA}$ & $\mathrm{UA} / \mathrm{NA}$ & EMORY \\
\hline 10 & $\mathrm{UA} / \mathrm{NA}$ & UA/NA & 10 & UA/NA & FLORIDA \\
\hline $\mathrm{UA} / \mathrm{NA}$ & UA/NA & UA/NA & UA/NA & UA/NA & FLORIDA STATE \\
\hline 1,173 & 0 & 1,169 & 4 & 0 & GEORGE WASHINGTON \\
\hline 1,008 & $\mathrm{UA} / \mathrm{NA}$ & $\mathrm{UA} / \mathrm{NA}$ & 1,008 & $\mathrm{UA} / \mathrm{NA}$ & GEORGETOWN \\
\hline $\mathrm{UA} / \mathrm{NA}$ & $\mathrm{UA} / \mathrm{NA}$ & $\mathrm{UA} / \mathrm{NA}$ & UA/NA & $\mathrm{UA} / \mathrm{NA}$ & HARVARD \\
\hline 3,361 & 0 & 3,242 & 94 & 25 & HAWAII \\
\hline 5,506 & 221 & 5,285 & UA/NA & UA/NA & HOWARD \\
\hline 20 & $\mathrm{UA} / \mathrm{NA}$ & $\mathrm{UA} / \mathrm{NA}$ & 20 & UA/NA & ILLINOIS, CHICAGO \\
\hline 5,382 & 4,231 & 1,138 & 13 & 0 & INDIANA \\
\hline 2,381 & 0 & 2,346 & 35 & $\mathrm{UA} / \mathrm{NA}$ & IOWA \\
\hline 37 & UA/NA & 37 & UA/NA & UA/NA & KANSAS \\
\hline 10 & UA/NA & UA/NA & 10 & UA/NA & KENTUCKY \\
\hline 10 & 0 & 0 & 10 & 0 & LOUISIANA STATE \\
\hline 703 & UA/NA & 276 & 427 & $\mathrm{UA} / \mathrm{NA}$ & MCGILL \\
\hline 17 & UA/NA & UA/NA & 17 & UA/NA & MCMASTER \\
\hline 81 & 0 & 0 & 81 & 0 & MIAMI \\
\hline 382 & 0 & 221 & 161 & 0 & MICHIGAN \\
\hline UA/NA & UA/NA & $\mathrm{UA} / \mathrm{NA}$ & UA/NA & UA/NA & MINNESOTA \\
\hline 46 & 0 & 0 & 46 & 0 & MONTREAL \\
\hline 48 & $\mathrm{UA} / \mathrm{NA}$ & 42 & 6 & 0 & NEW MEXICO \\
\hline $\mathrm{UA} / \mathrm{NA}$ & UA/NA & UA/NA & UA/NA & UA/NA & NEW YORK \\
\hline 1,067 & 0 & 979 & 88 & 0 & NORTH CAROLINA \\
\hline UA/NA & UA/NA & UA/NA & UA/NA & UA/NA & NORTHWESTERN \\
\hline 3,260 & 2,868 & 98 & 294 & 0 & OHIO STATE \\
\hline $\mathrm{UA} / \mathrm{NA}$ & $\mathrm{UA} / \mathrm{NA}$ & $\mathrm{UA} / \mathrm{NA}$ & $\mathrm{UA} / \mathrm{NA}$ & UA/NA & OKLAHOMA \\
\hline $\mathrm{UA} / \mathrm{NA}$ & $\mathrm{UA} / \mathrm{NA}$ & $\mathrm{UA} / \mathrm{NA}$ & UA/NA & UA/NA & OKLAHOMA STATE \\
\hline
\end{tabular}




\section{Collections Part 2}

\section{Current Serials}

Total

(5)
Current Serials

Purchased

(Survey Question Number)

INSTITUTION

PENNSYLVANIA

Notes

PENNSYLVANIA STATE

QUEEN'S

ROCHESTER

SASKATCHEWAN

SOUTH CAROLINA

SOUTHERN CALIFORNIA

SOUTHERN ILLINOIS

SUNY-STONY BROOK

TEMPLE

TENNESSEE

TEXAS TECH

TULANE

UTAH

VANDERBILT

VIRGINIA

WASHINGTON

WASHINGTON U.-ST. LOUIS

WISCONSIN

YALE

$\mathrm{b}$
$\mathrm{b}$
$\mathrm{b}$
$\mathrm{b}+$

\begin{tabular}{|c|c|c|c|c|}
\hline G & 4,922 & 4,922 & 4,878 & 44 \\
\hline$b+$ & 7,262 & 5,250 & 5,245 & 5 \\
\hline+ & UA/NA & UA/NA & UA/NA & UA/NA \\
\hline bG+ & 124 & 124 & UA/NA & 124 \\
\hline \multirow[t]{2}{*}{+} & 5,530 & 4,840 & 4,735 & 105 \\
\hline & 30,485 & 3,473 & 3,445 & 28 \\
\hline \multirow[t]{2}{*}{ G+ } & 2,749 & 2,029 & 1,848 & 181 \\
\hline & 1,152 & 1,152 & 521 & 631 \\
\hline b & 4,483 & 1,380 & 1,273 & 107 \\
\hline$b+$ & 7,598 & 5,158 & 4,840 & 318 \\
\hline bG+ & 1,787 & 936 & 752 & 184 \\
\hline $\mathrm{b}+$ & 22,483 & 20,169 & 19,735 & 434 \\
\hline \multirow{3}{*}{ bG } & 4,000 & 2,751 & 2,651 & 100 \\
\hline & 3,497 & 1,537 & 1,437 & 100 \\
\hline & 4,410 & 3,156 & 3,113 & 43 \\
\hline+ & $\mathrm{UA} / \mathrm{NA}$ & UA/NA & UA/NA & UA/NA \\
\hline+ & 0 & 0 & UA/NA & UA/NA \\
\hline bG+ & 4,012 & 2,846 & 2,820 & 26 \\
\hline $\mathrm{bG}^{+}$ & 4,740 & 1,108 & 1,024 & 84 \\
\hline G+ & 10,407 & 10,118 & 9,618 & 500 \\
\hline
\end{tabular}

(5a)
Current Serials
Purchased
Electronic

Current Serials

Purchased Print

(5a.ii)

(5a.i)

\section{Summary Data}

\section{MeAn}

MEdian

HigH

Low

TOTALS

Number of Libraries Reporting

+- See footnotes

UA/NA - Unavailable or Not Applicable

$\begin{array}{rr}6,628 & 4,316 \\ 4,586 & 2,038 \\ 30,971 & 30,589 \\ 91 & 91 \\ 331,408 & 211,471 \\ 50 & 49\end{array}$

G - Government documents not included in serials count

$\mathrm{b}$ - Basis of volume count is bibliographic

$\begin{array}{rr}4,561 & 309 \\ 2,820 & 142 \\ 29,880 & 2,041 \\ 97 & 5 \\ 186,985 & 14,842 \\ 41 & 48\end{array}$

. - no data supplied 


\section{Collections Part 2}

Current Serials

Not Purchased

(5b)
Current Serials

Not Purchased Consortial
Current Serials Not Purchased Freely Accessible
Current Serials

Not Purchased Print

(5b.iii)

Current Serials

Not Purchased GovDocs

(5b.iv) (Survey Question Number)

\begin{tabular}{|c|c|c|c|c|c|}
\hline & & & & & INSTITUTION \\
\hline $\mathrm{UA} / \mathrm{NA}$ & UA/NA & UA/NA & UA/NA & UA/NA & PENNSYLVANIA \\
\hline 2,012 & 1,005 & 1,007 & 0 & 0 & PENNSYLVANIA STATE \\
\hline UA/NA & UA/NA & UA/NA & UA/NA & UA/NA & QUEEN'S \\
\hline UA/NA & UA/NA & UA/NA & UA/NA & UA/NA & ROCHESTER \\
\hline 690 & 0 & 671 & 19 & 0 & SASKATCHEWAN \\
\hline 27,012 & 26,231 & 781 & 0 & 0 & SOUTH CAROLINA \\
\hline 720 & 0 & 720 & 0 & 0 & SOUTHERN CALIFORNIA \\
\hline UA/NA & UA/NA & UA/NA & UA/NA & UA/NA & SOUTHERN ILLINOIS \\
\hline 3,103 & 1,726 & 1,375 & 2 & 0 & SUNY-STONY BROOK \\
\hline 2,440 & 0 & 2,362 & 0 & 78 & TEMPLE \\
\hline 851 & 666 & 160 & 3 & 22 & TENNESSEE \\
\hline 2,314 & UA/NA & 2,270 & 44 & UA/NA & TEXAS TECH \\
\hline 1,249 & 0 & 1,249 & 0 & 0 & TULANE \\
\hline 1,960 & 1,718 & 224 & 10 & 8 & UTAH \\
\hline 1,254 & 527 & 723 & 0 & 4 & VANDERBILT \\
\hline $\mathrm{UA} / \mathrm{NA}$ & $\mathrm{UA} / \mathrm{NA}$ & $\mathrm{UA} / \mathrm{NA}$ & UA/NA & UA/NA & VIRGINIA \\
\hline 0 & $\mathrm{UA} / \mathrm{NA}$ & UA/NA & UA/NA & UA/NA & WASHINGTON \\
\hline 1,166 & 469 & 697 & UA/NA & UA/NA & WASHINGTON U.-ST. LOUIS \\
\hline 3,632 & UA/NA & 3,624 & 8 & 0 & WISCONSIN \\
\hline 289 & 0 & 200 & 89 & 0 & YALE \\
\hline \multicolumn{6}{|c|}{ Summary Data } \\
\hline 2,568 & 4,406 & 1,223 & 93 & 274 & MEAN \\
\hline 1,249 & 1,718 & 795 & 18 & 25 & Median \\
\hline 27,012 & 26,231 & 5,285 & 1,008 & 1,684 & HigH \\
\hline 10 & 14 & 37 & 2 & 4 & Low \\
\hline 110,437 & 57,278 & 41,585 & 2,608 & 1,917 & TOTALS \\
\hline 43 & 13 & 34 & 28 & 7 & Number of LibraRIES REPORTING \\
\hline
\end{tabular}




\section{Collections Part 3}

Microform Government Units Documents

(Survey Question Number)

(7)

(8)
Computer Manuscripts Cartographic

Files and Archives Materials
Graphic

(9) (10)

(11)

Materials

Audio

Materials

Film and

Notes

\begin{tabular}{|c|c|c|c|c|c|c|c|c|c|}
\hline ALABAMA & $\mathrm{bG}+$ & 0 & 0 & 125 & 0 & 0 & 1,546 & 498 & 357 \\
\hline ARIZONA & bG+ & 4,700 & 0 & 0 & 15 & 34 & 71,719 & 596 & 6,650 \\
\hline BOSTON & & 29 & 0 & 366 & 751 & 0 & 0 & 17 & 112 \\
\hline CALIFORNIA, DAVIS & $\mathrm{b}+$ & 23,780 & 0 & 305 & 0 & 0 & 70 & 1,338 & 349 \\
\hline CALIFORNIA, IRVINE & bG & 349,627 & 17,892 & 1,453 & 540 & 1,369 & 8,599 & 1,997 & 1,846 \\
\hline CALIFORNIA, LOS ANGELES & bG+ & 64,852 & 0 & 2,226 & 1,578 & 3 & 43,987 & 3,569 & 1,660 \\
\hline CALIFORNIA, SAN DIEGO & bG+ + & 1,483 & 0 & 834 & 0 & 0 & 0 & 2,032 & 7,222 \\
\hline CASE WESTERN RESERVE & $\mathrm{G}+$ & 3,752 & 0 & 500 & 1,000 & 0 & 51,833 & 1,300 & 1,321 \\
\hline CHICAGO & G+ & 29,108 & 0 & 1,573 & 0 & 0 & 0 & UA/NA & $\mathrm{UA} / \mathrm{NA}$ \\
\hline CINCINNATI & $\mathrm{b}+$ & 0 & 0 & 0 & 3,070 & 0 & 6,000 & 2,140 & 849 \\
\hline COLUMBIA & + & 52,822 & UA/NA & 957 & 4,407 & UA/NA & 13,474 & UA/NA & 1,112 \\
\hline CONNECTICUT & G+ & 0 & 0 & 460 & 0 & 0 & 0 & 56 & 1,023 \\
\hline CORNELL & + & 12,900 & 0 & 612 & 0 & 0 & 900 & 29 & 954 \\
\hline DARTMOUTH & bG+ & 13,059 & UA/NA & 421 & UA/NA & 3 & 20,313 & 5,824 & 1,467 \\
\hline DUKE & $\mathrm{b}$ & 193 & 0 & 30,492 & 11,911 & 93 & 555,689 & 1,260 & 2,965 \\
\hline EMORY & + & 15 & . & 198 & 257 & UA/NA & UA/NA & 4,304 & 1,475 \\
\hline FLORIDA & + & 1,747 & 0 & 66 & . & . & . & -267 & -142 \\
\hline FLORIDA STATE & $\mathrm{b}+$ & 0 & 0 & 0 & 0 & 0 & 0 & 0 & 103 \\
\hline GEORGE WASHINGTON & bG+ & 0 & 0 & 365 & 75 & 0 & 3,656 & 836 & 1,183 \\
\hline GEORGETOWN & $\mathrm{b}+$ & 0 & 0 & 61 & 0 & 0 & 69 & 0 & 105 \\
\hline HARVARD & & 2,032 & . & . & . & . & . & . & \\
\hline HAWAII & $\mathrm{b}+$ & 0 & 0 & 104 & 0 & 0 & 0 & 1 & 0 \\
\hline HOWARD & $\mathrm{bG}$ & 20,000 & 0 & 0 & 0 & 0 & 0 & 65 & 1,520 \\
\hline ILLINOIS, CHICAGO & + & 61,616 & 0 & 1,848 & UA/NA & $\mathrm{UA} / \mathrm{NA}$ & UA/NA & 1,260 & 2,368 \\
\hline INDIANA & bG+ & 759 & 0 & 652 & 130 & 0 & 0 & 152 & 297 \\
\hline IOWA & $\mathrm{b}+$ & UA/NA & UA/NA & UA/NA & UA/NA & UA/NA & UA/NA & 219 & 488 \\
\hline KANSAS & + & . & . & 1,518 & . & . & 153 & 62 & 1,281 \\
\hline KENTUCKY & G+ & 2 & 0 & 14 & 0 & 0 & 31,598 & 1,274 & 1,395 \\
\hline LOUISIANA STATE & + & 0 & 0 & 324 & 0 & 0 & 0 & 0 & 716 \\
\hline MCGILL & $\mathrm{bG}+$ & 11,523 & 0 & 1,319 & 252 & 0 & 8,345 & 174 & 6,339 \\
\hline MCMASTER & $\mathrm{b}+$ & 5,241 & 0 & 2,086 & 855 & 0 & 10 & 867 & 891 \\
\hline MIAMI & $\mathrm{G}+$ & 2,837 & 0 & $\mathrm{UA} / \mathrm{NA}$ & 610 & $\mathrm{UA} / \mathrm{NA}$ & $\mathrm{UA} / \mathrm{NA}$ & $\mathrm{UA} / \mathrm{NA}$ & 1,753 \\
\hline MICHIGAN & bG+ & 3,966 & 0 & 1,399 & 3 & 0 & 0 & 0 & 12 \\
\hline MINNESOTA & $\mathrm{b}+$ & 67,186 & 19,979 & 645 & 80 & 9 & UA/NA & UA/NA & $\mathrm{UA} / \mathrm{NA}$ \\
\hline MONTREAL & $\mathrm{G}^{+}$ & 31,540 & 0 & 1,118 & 0 & 0 & 12,727 & 998 & 1,770 \\
\hline NEW MEXICO & + & 2 & 0 & 456 & 1,896 & 0 & 7,427 & 1,281 & 2,126 \\
\hline NEW YORK & bG+ & 4 & 0 & 670 & 707 & 0 & 8,861 & 336 & 1,344 \\
\hline NORTH CAROLINA & G & 35,888 & 0 & 19 & 0 & 19 & 820 & 131 & 2,308 \\
\hline NORTHWESTERN & G+ & 0 & 0 & 712 & . & 0 & . & 478 & 1,143 \\
\hline OHIO STATE & bG & 102 & 0 & 537 & 1,134 & 1,670 & 10,000 & 112 & 1,271 \\
\hline OKLAHOMA & + & 650 & . & 132 & 5,921 & . & . & . & 6,502 \\
\hline OKLAHOMA STATE & + & 44,260 & 0 & 16 & 168 & 2 & 3,844 & 3,432 & 1,586 \\
\hline
\end{tabular}




\section{Collections Part 3}

\begin{tabular}{rrrrrrrrr} 
& Microform & Government & Computer & Manuscripts Cartographic & Graphic & \multicolumn{2}{c}{ Audio } & Film and \\
Units & Documents & Files and Archives & Materials & Materials & Materials & Video \\
(Survey Question Number) & $(7)$ & $(8)$ & $(9)$ & $(10)$ & $(11)$ & $(12)$ & (13) & (14)
\end{tabular}

INSTITUTION

\begin{tabular}{|c|c|c|c|c|c|c|c|c|c|}
\hline PENNSYLVANIA & G & UA/NA & UA/NA & UA/NA & UA/NA & UA/NA & UA/NA & UA/NA & UA/NA \\
\hline PENNSYLVANIA STATE & $\mathrm{b}+$ & 0 & 0 & 285 & 93 & 0 & 743 & 0 & 387 \\
\hline QUEEN'S & + & UA/NA & UA/NA & UA/NA & UA/NA & UA/NA & UA/NA & $\mathrm{UA} / \mathrm{NA}$ & $\mathrm{UA} / \mathrm{NA}$ \\
\hline ROCHESTER & $\mathrm{bG}+$ & 0 & 0 & 21 & 1,316 & 0 & 5,000 & 65 & 77 \\
\hline SASKATCHEWAN & + & 2,290 & 0 & 78 & 0 & 0 & 10,986 & 169 & 47 \\
\hline SOUTH CAROLINA & & 4,721 & 0 & 9 & 0 & 0 & 0 & 81 & 1,411 \\
\hline SOUTHERN CALIFORNIA & G+ & 8,567 & 0 & 707 & 35 & 0 & 12,591 & 2,283 & 644 \\
\hline SOUTHERN ILLINOIS & & 6,111 & 338 & 85 & 410 & 0 & 0 & 2,574 & 3,436 \\
\hline SUNY-STONY BROOK & $\mathrm{b}$ & 0 & 0 & 0 & 0 & 0 & 0 & 0 & 0 \\
\hline TEMPLE & $b+$ & 49 & 0 & 18 & 0 & 0 & 0 & 0 & 1,513 \\
\hline TENNESSEE & $\mathrm{bG}+$ & 328 & 0 & 313 & 205 & 0 & 0 & 8 & 760 \\
\hline TEXAS TECH & $b+$ & 2,550 & 0 & 2,567 & 750 & 0 & 786 & 5,037 & 7,493 \\
\hline TULANE & & 0 & 0 & 290 & 1,351 & 0 & 0 & 0 & 0 \\
\hline UTAH & $\mathrm{bG}$ & 15,401 & 0 & 884 & 0 & 0 & 1,970 & 10 & 1,015 \\
\hline VANDERBILT & & 9,119 & 0 & 1,083 & 3,924 & 2 & 0 & 1,272 & 1,352 \\
\hline VIRGINIA & + & 42 & 0 & 280 & 859 & 0 & 0 & 6 & 1,863 \\
\hline WASHINGTON & + & 85,211 & . & 3,065 & . & . & 454 & . & 827 \\
\hline WASHINGTON U.-ST. LOUIS & $\mathrm{bG}+$ & 1,854 & 0 & 903 & 2,495 & . & 3 & 25 & 731 \\
\hline WISCONSIN & $\mathrm{bG}+$ & 6,567 & 6,303 & 75 & 3,620 & 0 & 11 & 681 & 1,346 \\
\hline YALE & G+ & 15,651 & 0 & 819 & 532 & 0 & 11,235 & 2,310 & 730 \\
\hline \multicolumn{10}{|c|}{ Summary DATA } \\
\hline MEAN & & 22,314 & 11,128 & 1,270 & 1,544 & 320 & 27,437 & 1,131 & 1,655 \\
\hline MEDian & & 4,721 & 12,098 & 480 & 750 & 14 & 6,000 & 596 & 1,276 \\
\hline HiGH & & 349,627 & 19,979 & 30,492 & 11,911 & 1,670 & 555,689 & 5,824 & 7,493 \\
\hline Low & & 2 & 338 & 9 & 3 & 2 & 3 & -267 & -142 \\
\hline TOTAls & & $1,004,136$ & 44,512 & 66,065 & 50,950 & 3,204 & 905,419 & 50,892 & 89,353 \\
\hline NuMBER OF LIBRARIES REPORTII & & 45 & 4 & 52 & 33 & 10 & 33 & 45 & 54 \\
\hline
\end{tabular}

+- See footnotes

UA/NA - Unavailable or Not Applicable
G - Government documents not included in serials count $\mathrm{b}$ - Basis of volume count is bibliographic

- - no data supplied 


\section{EXPENDITURES}

Monographs Current Serials Other Library Miscellaneous Total Library

Contract Materials Materials Materials

(16a)

(16b)

(16c)

(16d)

(16)

INSTITUTION

ALABAMA
ARIZONA
BOSTON

CALIFORNIA, DAVIS

CALIFORNIA, IRVINE

CALIFORNIA, LOS ANGELES

CALIFORNIA, SAN DIEGO

CASE WESTERN RESERVE

CHICAGO

CINCINNATI

COLUMBIA

CONNECTICUT

CORNELL

DARTMOUTH

DUKE

EMORY

FLORIDA

FLORIDA STATE

GEORGE WASHINGTON

GEORGETOWN

HARVARD

HAWAII

HOWARD

ILLINOIS, CHICAGO

INDIANA

IOWA

KANSAS

KENTUCKY

LOUISIANA STATE

MCGILL

MCMASTER

MIAMI

MICHIGAN

MINNESOTA

MONTREAL

NEW MEXICO

NEW YORK

NORTH CAROLINA

NORTHWESTERN

OHIO STATE

OKLAHOMA

OKLAHOMA STATE

\section{Notes}

b

bG+ 5,064

bG+ $\quad 10,873$

35,827

273,044

bG

bG+

bG+

G+

G+

b+

$+$

G+

$+\quad 145,483$

bG+

b

b

$+$

$+$

$+$

b+

bG+

b+

b+

bG+

$+$

$+$

bG+

b+

$+$

$+$

G+

$+$

bG+

b+

G+

bG+

b+

G+

$+$

bG+

G

G+

bG

$+$

$+$

(2)

\section{9,254}

54,106

95,042

40,102

110,017

\section{6,707}

39,000

90,253

91,657

40,000

387,468

234,117

80,960

119,231

179,161

19,466

470,705

113,127

126,818

164,648

452,368

290,645

130,308

95,000

149,130

88,676

70,403

163,631

45,310

16 - ARL Academic Health Sciences Library Statistics 2008-2009 


\section{EXPENDITURES}

Salaries \& Wages Professional Staff*

(18a)
Salaries \& Wages Salaries \& Wages Total Salaries \& Other Operating Support Staff* Student Assistants*

$(18 b)$

(18c)

(18)
Total Library

Expenditures

(21) (Survey Question Number)

\begin{tabular}{|c|c|c|c|c|c|c|}
\hline 153,577 & 56,464 & 870 & 210,911 & 11,720 & 359,925 & ALABAMA \\
\hline $1,024,835$ & 534,347 & 73,830 & $1,633,012$ & 748,993 & $3,797,023$ & ARIZONA \\
\hline 751,390 & 318,045 & 63,061 & $1,132,496$ & 99,638 & $2,750,466$ & BOSTON \\
\hline 627,694 & 405,527 & 63,485 & $1,096,706$ & 15,163 & $2,655,911$ & CALIFORNIA, DAVIS \\
\hline 537,108 & 608,215 & 76,715 & $1,222,038$ & 266,987 & $2,633,748$ & CALIFORNIA, IRVINE \\
\hline $1,157,135$ & 542,449 & 278,910 & $1,978,494$ & 635,272 & $4,967,481$ & CALIFORNIA, LOS ANGELES \\
\hline 702,487 & 686,057 & 120,703 & $1,509,247$ & 50,051 & $3,665,036$ & CALIFORNIA, SAN DIEGO \\
\hline 488,185 & 357,545 & 143,531 & 989,261 & 325,396 & $2,943,992$ & CASE WESTERN RESERVE \\
\hline 305,653 & 481,981 & 118,117 & 905,751 & 34,514 & $5,343,542$ & CHICAGO \\
\hline 991,902 & 114,175 & 60,594 & $1,166,671$ & 460,517 & $3,391,477$ & CINCINNATI \\
\hline 937,040 & 373,908 & 9,656 & $1,320,604$ & 70,998 & $3,847,309$ & COLUMBIA \\
\hline $1,145,595$ & 686,890 & 50,303 & $1,882,788$ & 70,009 & $4,092,075$ & CONNECTICUT \\
\hline 600,702 & $1,269,686$ & 0 & $1,870,388$ & 189,661 & $3,563,377$ & CORNELL \\
\hline 554,349 & 505,844 & 26,650 & $1,086,843$ & 151,451 & $2,422,847$ & DARTMOUTH \\
\hline $1,209,935$ & 685,397 & 0 & $1,895,332$ & 435,512 & $4,026,532$ & DUKE \\
\hline 780,922 & 877,768 & 12,406 & $1,671,096$ & 643,668 & $4,817,241$ & EMORY \\
\hline $1,312,110$ & 967,045 & 102,408 & $2,381,563$ & 422,564 & $4,692,615$ & FLORIDA \\
\hline 256,099 & 51,900 & 28,980 & 336,979 & 28,392 & $1,224,938$ & FLORIDA STATE \\
\hline 839,460 & 630,755 & 107,643 & $1,577,858$ & 171,895 & $3,950,880$ & GEORGE WASHINGTON \\
\hline 693,463 & 345,479 & 40,000 & $1,078,942$ & 143,440 & $3,092,083$ & GEORGETOWN \\
\hline $2,760,609$ & 764,701 & 7,657 & $3,532,967$ & 525,324 & $6,533,719$ & HARVARD \\
\hline 219,952 & 135,796 & $\mathrm{UA} / \mathrm{NA}$ & 355,748 & 15,610 & 827,284 & HAWAII \\
\hline 470,699 & 303,140 & 125,000 & 898,839 & 12,000 & $2,117,244$ & HOWARD \\
\hline 838,277 & $1,222,749$ & 79,613 & $2,140,639$ & 125,678 & $4,954,663$ & ILLINOIS, CHICAGO \\
\hline 843,614 & 759,908 & 48,648 & $1,652,170$ & 188,288 & $4,636,115$ & INDIANA \\
\hline 631,713 & 478,375 & 164,374 & $1,274,462$ & 91,872 & $2,568,927$ & IOWA \\
\hline 517,992 & 797,143 & 53,832 & $1,368,967$ & 281,878 & $3,377,400$ & KANSAS \\
\hline 803,850 & 281,655 & 83,398 & $1,168,903$ & 167,992 & $3,350,411$ & KENTUCKY \\
\hline 45,910 & 122,500 & 1,550 & 169,960 & 20,280 & 619,349 & LOUISIANA STATE \\
\hline 618,385 & 395,461 & 27,440 & $1,041,286$ & 81,341 & $2,797,751$ & MCGILL \\
\hline 461,213 & 685,329 & 67,636 & $1,214,178$ & 124,174 & $2,645,078$ & MCMASTER \\
\hline $1,006,358$ & 762,939 & 0 & $1,769,297$ & 433,694 & $4,344,461$ & MIAMI \\
\hline 914,570 & 746,830 & 207,650 & $1,869,050$ & 18,754 & $3,824,455$ & MICHIGAN \\
\hline 779,776 & $1,115,197$ & 205,606 & $2,100,579$ & 731,442 & $6,239,579$ & MINNESOTA \\
\hline 618,359 & 931,701 & 0 & $1,550,060$ & 43,557 & $3,782,830$ & MONTREAL \\
\hline $1,125,494$ & 754,867 & 30,000 & $1,910,361$ & 224,169 & $3,349,138$ & NEW MEXICO \\
\hline $1,832,523$ & $1,224,926$ & 67,660 & $3,125,109$ & 461,229 & $6,003,589$ & NEW YORK \\
\hline $2,269,841$ & $1,341,009$ & 162,697 & $3,773,547$ & 547,703 & $7,770,531$ & NORTH CAROLINA \\
\hline $1,114,793$ & 457,444 & 11,880 & $1,584,117$ & 240,164 & $4,143,803$ & NORTHWESTERN \\
\hline $2,757,211$ & 688,012 & 202,596 & $3,647,819$ & 439,308 & $5,685,776$ & OHIO STATE \\
\hline 496,713 & 650,246 & 128,914 & $1,275,873$ & 623,510 & $4,095,123$ & OKLAHOMA \\
\hline 207,307 & 342,551 & 66,390 & 616,248 & 232,742 & $1,219,764$ & OKLAHOMA STATE \\
\hline
\end{tabular}




\section{EXPENDITURES}

Monographs Current Serials Other Library Miscellaneous Total Library

Contract Materials Materials Materials

(16b) (16c) (16d)
Binding

(17)

\begin{tabular}{|c|c|c|c|c|c|c|c|}
\hline INSTITUTION & Notes & & & & & & \\
\hline PENNSYLVANIA & G & 133,265 & $2,166,113$ & UA/NA & UA/NA & $2,299,378$ & 1,583 \\
\hline PENNSYLVANIA STATE & $\mathrm{b}+$ & 22,060 & $1,536,436$ & 117,315 & 0 & $1,675,811$ & 159 \\
\hline QUEEN'S & + & 109,261 & $1,381,325$ & 9,693 & 0 & $1,500,279$ & 3,359 \\
\hline ROCHESTER & bG+ & 70,516 & $1,887,468$ & 0 & 0 & $1,957,984$ & 13,229 \\
\hline SASKATCHEWAN & + & 653,614 & $2,024,209$ & 0 & UA/NA & $2,677,823$ & 4,695 \\
\hline SOUTH CAROLINA & & 15,827 & 498,849 & 126,716 & 0 & 641,392 & 0 \\
\hline SOUTHERN CALIFORNIA & G+ & 331,885 & $2,351,067$ & 7,696 & 276,450 & $2,967,098$ & 34,662 \\
\hline SOUTHERN ILLINOIS & & 76,108 & 732,828 & 0 & 38,251 & 847,187 & 13,887 \\
\hline SUNY-STONY BROOK & $\mathrm{b}+$ & 57,285 & $1,476,610$ & 140,333 & 8,195 & $1,682,423$ & 0 \\
\hline TEMPLE & $\mathrm{b}+$ & 210,717 & $1,693,415$ & 567,148 & 7,494 & $2,478,774$ & 8,416 \\
\hline TENNESSEE & bG+ & 46,554 & $1,744,948$ & 53,768 & 4,860 & $1,850,130$ & 4,755 \\
\hline TEXAS TECH & $\mathrm{b}+$ & 281,735 & $1,878,860$ & 51,890 & 421,378 & $2,633,863$ & 14,305 \\
\hline TULANE & & 92,793 & $1,114,393$ & 70,293 & 0 & $1,277,479$ & 0 \\
\hline UTAH & bG & 109,212 & 720,817 & 122,336 & 6,373 & 958,738 & 6,496 \\
\hline VANDERBILT & & 210,010 & $2,610,628$ & 46,305 & 39,800 & $2,906,743$ & 0 \\
\hline VIRGINIA & + & 159,844 & $1,509,651$ & $1,511,581$ & 3,761 & $3,184,837$ & 2,108 \\
\hline WASHINGTON & + & 120,807 & $2,666,858$ & . & 5,012 & $2,792,677$ & 9,397 \\
\hline WASHINGTON U.-ST. LOUIS & bG+ & 252,128 & $3,030,772$ & 0 & 0 & $3,282,900$ & 24,998 \\
\hline WISCONSIN & bG+ & 38,610 & $1,176,374$ & 664 & 285,932 & $1,501,580$ & 29,759 \\
\hline YALE & G+ & 675,136 & $2,057,873$ & 38,406 & 0 & $2,771,415$ & 9,275 \\
\hline \multicolumn{8}{|c|}{ SuMMARY DATA } \\
\hline MeAn & & 161,291 & $1,495,939$ & 262,503 & 97,077 & $1,918,566$ & 13,271 \\
\hline MEdian & & 116,179 & $1,502,805$ & 53,768 & 19,310 & $1,878,193$ & 8,132 \\
\hline HigH & & 675,136 & $3,030,772$ & $3,141,418$ & 458,047 & $4,350,544$ & 67,835 \\
\hline Low & & 5,064 & 101,364 & 314 & 350 & 135,888 & 159 \\
\hline TOTAls & & $10,000,021$ & $92,748,218$ & $13,387,661$ & $2,815,221$ & $118,951,121$ & 650,255 \\
\hline Number of Libraries Reporting & & 62 & 62 & 51 & 29 & 62 & 49 \\
\hline
\end{tabular}

+- See footnotes

G-Government documents not included in serials count

- - no data supplied

UA/NA - Unavailable or Not Applicable

$\mathrm{b}$ - Basis of volume count is bibliographic 


\section{EXPENDITURES}

Salaries \& Wages Professional Staff ${ }^{\star}$

(18a)

Salaries \& Wages Salaries \& Wages Total Salaries \& Support Staff* Student Assistants*

(18b)

(18c)
Wages

(18) perating Expenditures

(20)
Total Library Expenditures

(21) (Survey Question Number)

\begin{tabular}{|c|c|c|c|c|c|c|}
\hline & & & & & & INSTITUTION \\
\hline 471,479 & 545,187 & 116,582 & $1,133,248$ & 327,874 & $3,762,083$ & PENNSYLVANIA \\
\hline 433,152 & 346,262 & 63,234 & 842,648 & 72,303 & $2,590,921$ & PENNSYLVANIA STATE \\
\hline 559,798 & 414,459 & 31,137 & $1,005,394$ & 163,606 & $2,672,638$ & QUEEN'S \\
\hline $1,276,556$ & 208,708 & 55,547 & $1,540,811$ & 344,731 & $3,856,755$ & ROCHESTER \\
\hline 474,731 & 221,041 & 51,867 & 747,639 & $\mathrm{UA} / \mathrm{NA}$ & $3,430,158$ & SASKATCHEWAN \\
\hline 441,431 & 222,789 & 20,762 & 684,982 & 72,166 & $1,398,540$ & SOUTH CAROLINA \\
\hline 939,966 & 992,082 & 138,828 & $2,070,876$ & 667,400 & $5,740,036$ & SOUTHERN CALIFORNIA \\
\hline 430,108 & 452,794 & 13,743 & 896,645 & 81,620 & $1,839,339$ & SOUTHERN ILLINOIS \\
\hline $1,240,391$ & 261,305 & 138,476 & $1,640,172$ & 469,237 & $3,791,832$ & SUNY-STONY BROOK \\
\hline 701,329 & 458,188 & 77,156 & $1,236,673$ & 470,130 & $4,193,993$ & TEMPLE \\
\hline 950,564 & 528,390 & 13,199 & $1,492,153$ & 140,791 & $3,487,829$ & TENNESSEE \\
\hline $1,119,666$ & 730,802 & 245,350 & $2,095,818$ & 309,820 & $5,053,806$ & TEXAS TECH \\
\hline 384,474 & 177,413 & 13,224 & 575,111 & 48,088 & $1,900,678$ & TULANE \\
\hline 775,376 & 852,617 & 68,821 & $1,696,814$ & $1,364,647$ & $4,026,695$ & UTAH \\
\hline $1,724,832$ & 486,926 & 28,403 & $2,240,161$ & 742,692 & $5,889,596$ & VANDERBILT \\
\hline $1,009,521$ & 809,111 & 8,546 & $1,827,178$ & 272,657 & $5,286,780$ & VIRGINIA \\
\hline $1,856,841$ & 705,848 & 170,976 & $2,733,665$ & $2,604,951$ & $8,140,690$ & WASHINGTON \\
\hline $1,956,083$ & 652,861 & 34,412 & $2,643,356$ & 192,155 & $6,143,409$ & WASHINGTON U.-ST. LOUIS \\
\hline $1,022,589$ & 534,986 & 101,785 & $1,659,360$ & 775,320 & $3,966,019$ & WISCONSIN \\
\hline $1,586,523$ & 766,735 & 35,759 & $2,389,017$ & 354,033 & $5,523,740$ & YALE \\
\hline \multicolumn{7}{|c|}{ SUMMARY DATA } \\
\hline 899,358 & 577,911 & 79,793 & $1,550,627$ & 329,193 & $3,803,565$ & MEAN \\
\hline 780,349 & 538,718 & 63,485 & $1,525,029$ & 224,169 & $3,794,428$ & Median \\
\hline $2,760,609$ & $1,341,009$ & 278,910 & $3,773,547$ & $2,604,951$ & $8,140,690$ & $\mathrm{HigH}$ \\
\hline 45,910 & 51,900 & 870 & 169,960 & 11,720 & 359,925 & Low \\
\hline $55,760,211$ & $35,830,459$ & $4,548,210$ & $96,138,880$ & $20,080,771$ & $235,821,026$ & TOTALS \\
\hline 62 & 62 & 57 & 62 & 61 & 62 & NUMBER OF LibraRIES REPORTING \\
\hline
\end{tabular}

${ }^{*}$ Figures in italics indicate the inclusion of fringe benefits. 


\section{Electronic Resources Expenditures}

One-time Electronic Resource Purchases
Ongoing Electronic Resource Purchases
Total

(22)
(23)
Electronic Resource Purchases
$(22+23)$
Total

Library Materials Expenditures
Electronic Resources as a \% of Library Materials Budget

(Survey Question Number)

(16)

\section{INSTITUTION}

\begin{tabular}{|c|c|c|c|c|c|c|}
\hline INSTITUTION & Notes & & & & & \\
\hline ALABAMA & $\mathrm{bG}+$ & 3,154 & 73,285 & 76,439 & 135,888 & 56.25 \\
\hline ARIZONA & $\mathrm{bG}+$ & 4,500 & $1,304,113$ & $1,308,613$ & $1,413,727$ & 92.56 \\
\hline BOSTON & & 24,008 & 946,147 & 970,155 & $1,515,436$ & 64.02 \\
\hline CALIFORNIA, DAVIS & $\mathrm{b}+$ & 0 & $1,131,891$ & $1,131,891$ & $1,544,042$ & 73.31 \\
\hline CALIFORNIA, IRVINE & bG & 66,501 & 580,290 & 646,791 & $1,138,740$ & 56.80 \\
\hline CALIFORNIA, LOS ANGELES & $\mathrm{bG}+$ & UA/NA & $\mathrm{UA} / \mathrm{NA}$ & . & $2,307,991$ & . \\
\hline CALIFORNIA, SAN DIEGO & $\mathrm{bG}+$ & 80,137 & $1,386,053$ & $1,466,190$ & $2,105,738$ & 69.63 \\
\hline CASE WESTERN RESERVE & $\mathrm{G}^{+}$ & 4,560 & $1,489,481$ & $1,494,041$ & $1,623,204$ & 92.04 \\
\hline CHICAGO & $\mathrm{G}^{+}$ & 86,512 & $3,006,852$ & $3,093,364$ & $4,350,544$ & 71.10 \\
\hline CINCINNATI & $\mathrm{b}+$ & 105,816 & $1,178,639$ & $1,284,455$ & $1,757,637$ & 73.08 \\
\hline COLUMBIA & + & UA/NA & $1,878,538$ & $1,878,538$ & $2,445,876$ & 76.80 \\
\hline CONNECTICUT & $\mathrm{G}^{+}$ & 10,168 & $1,629,172$ & $1,639,340$ & $2,128,814$ & 77.01 \\
\hline CORNELL & + & 138,229 & 945,642 & $1,083,871$ & $1,486,428$ & 72.92 \\
\hline DARTMOUTH & bG+ & 23,055 & $1,009,620$ & $1,032,675$ & $1,179,878$ & 87.52 \\
\hline DUKE & $\mathrm{b}$ & 0 & $1,216,650$ & $1,216,650$ & $1,691,910$ & 71.91 \\
\hline EMORY & + & 87,477 & $1,016,267$ & $1,103,744$ & $2,484,557$ & 44.42 \\
\hline FLORIDA & + & . & . & . & $1,888,488$ & . \\
\hline FLORIDA STATE & $b+$ & 14,651 & 839,423 & 854,074 & 859,567 & 99.36 \\
\hline GEORGE WASHINGTON & $\mathrm{bG}+$ & 67,139 & $1,534,909$ & $1,602,048$ & $2,192,995$ & 73.05 \\
\hline GEORGETOWN & $\mathrm{b}+$ & 0 & $1,612,000$ & $1,612,000$ & $1,867,897$ & 86.30 \\
\hline HARVARD & & $2,114,111$ & . & $2,114,111$ & $2,407,593$ & 87.81 \\
\hline HAWAII & $\mathrm{b}+$ & 0 & 405,404 & 405,404 & 455,926 & 88.92 \\
\hline HOWARD & $\mathrm{bG}+$ & 0 & $1,066,473$ & $1,066,473$ & $1,200,405$ & 88.84 \\
\hline ILLINOIS, CHICAGO & + & 22,003 & $1,508,822$ & $1,530,825$ & $2,656,181$ & 57.63 \\
\hline INDIANA & $\mathrm{bG}+$ & 913,330 & $1,813,477$ & $2,726,807$ & $2,795,102$ & 97.56 \\
\hline IOWA & $\mathrm{b}+$ & 135 & 851,293 & 851,428 & $1,202,593$ & 70.80 \\
\hline KANSAS & + & 77,031 & $1,100,000$ & $1,177,031$ & $1,719,603$ & 68.45 \\
\hline KENTUCKY & $\mathrm{G}^{+}$ & 18,720 & $1,415,260$ & $1,433,980$ & $2,006,594$ & 71.46 \\
\hline LOUISIANA STATE & + & 0 & 406,400 & 406,400 & 429,109 & 94.71 \\
\hline MCGILL & bG+ & 62,280 & $1,014,627$ & $1,076,907$ & $1,675,125$ & 64.29 \\
\hline MCMASTER & $b+$ & UA/NA & UA/NA & . & $1,305,141$ & \\
\hline MIAMI & $\mathrm{G}+$ & 2,890 & $1,666,661$ & $1,669,551$ & $2,134,363$ & 78.22 \\
\hline MICHIGAN & $\mathrm{bG}+$ & 177,369 & 636,949 & 814,318 & $1,936,651$ & 42.05 \\
\hline MINNESOTA & $\mathrm{b}+$ & 48,662 & $2,342,874$ & $2,391,536$ & $3,369,145$ & 70.98 \\
\hline MONTREAL & G+ & 47,723 & $1,685,019$ & $1,732,742$ & $2,179,373$ & 79.51 \\
\hline NEW MEXICO & + & 13,019 & $1,124,970$ & $1,137,989$ & $1,213,562$ & 93.77 \\
\hline NEW YORK & bG+ & 0 & $2,253,833$ & $2,253,833$ & $2,402,949$ & 93.79 \\
\hline NORTH CAROLINA & G & 86,536 & $2,990,712$ & $3,077,248$ & $3,433,967$ & 89.61 \\
\hline NORTHWESTERN & G+ & 121,229 & $2,035,503$ & $2,156,732$ & $2,303,404$ & 93.63 \\
\hline OHIO STATE & $\mathrm{bG}$ & 2,730 & 2,728 & 5,458 & $1,587,886$ & .34 \\
\hline OKLAHOMA & + & 12,435 & $1,489,611$ & $1,502,046$ & $2,158,716$ & 69.58 \\
\hline OKLAHOMA STATE & + & 9,651 & 259,639 & 269,290 & 369,865 & 72.81 \\
\hline
\end{tabular}

$20 \cdot$ ARL Academic Health Sciences Library Statistics 2008-2009 


\section{Electronic Resources ExPenditures}

Bibl. Utilities, Networks, etc. Bibl. Utilities, Networks, etc. Computer Hardware and Library Expenditures
External Expenditures

\section{Software Expenditures}

Document Delivery/
Interlibrary Loan Expenditures (24a)

(24b)

(26) (Survey Question Number)

\begin{tabular}{|c|c|c|c|c|}
\hline & & & & INSTITUTION \\
\hline 965 & 0 & 123 & 1,881 & ALABAMA \\
\hline 0 & 924,391 & 36,611 & 58,754 & ARIZONA \\
\hline 5,352 & . & 81,954 & 1,857 & BOSTON \\
\hline 76,317 & 0 & 13,648 & 42,124 & CALIFORNIA, DAVIS \\
\hline 16,622 & 88,641 & 68,689 & 8,067 & CALIFORNIA, IRVINE \\
\hline UA/NA & UA/NA & $\mathrm{UA} / \mathrm{NA}$ & $\mathrm{UA} / \mathrm{NA}$ & CALIFORNIA, LOS ANGELES \\
\hline UA/NA & UA/NA & 47,485 & 94,647 & CALIFORNIA, SAN DIEGO \\
\hline 9,744 & 0 & 4,013 & 11,040 & CASE WESTERN RESERVE \\
\hline UA/NA & UA/NA & 125,000 & 28,524 & CHICAGO \\
\hline 10,000 & 0 & 57,091 & 1,602 & CINCINNATI \\
\hline 8,955 & UA/NA & 97,435 & $\mathrm{UA} / \mathrm{NA}$ & COLUMBIA \\
\hline 20,616 & 0 & 58,042 & 9,313 & CONNECTICUT \\
\hline 17,266 & 0 & 140,018 & 7,098 & CORNELL \\
\hline UA/NA & UA/NA & 10,510 & 83,724 & DARTMOUTH \\
\hline 81,144 & 0 & 106,550 & 3,736 & DUKE \\
\hline 4,727 & 0 & 87,304 & 9,637 & EMORY \\
\hline 13,991 & . & 32,560 & 9,140 & FLORIDA \\
\hline 0 & 0 & 0 & 717 & FLORIDA STATE \\
\hline 4,757 & UA/NA & 71,341 & 7,647 & GEORGE WASHINGTON \\
\hline 3,377 & 0 & 43,121 & 4,374 & GEORGETOWN \\
\hline 9,555 & . & 79,521 & . & HARVARD \\
\hline 20,372 & 421 & 7,098 & UA/NA & HAWAII \\
\hline 0 & 4,500 & 30,000 & 8,500 & HOWARD \\
\hline UA/NA & $\mathrm{UA} / \mathrm{NA}$ & 36,799 & 32,594 & ILLINOIS, CHICAGO \\
\hline 0 & 0 & 0 & 0 & INDIANA \\
\hline 0 & 0 & 350 & 60,989 & IOWA \\
\hline & . & 75,798 & . & KANSAS \\
\hline 8,000 & 0 & 48,767 & 64,808 & KENTUCKY \\
\hline 0 & 0 & 0 & 2,920 & LOUISIANA STATE \\
\hline . & . & . & . & MCGILL \\
\hline UA/NA & UA/NA & 35,164 & 27,392 & MCMASTER \\
\hline 10,519 & 0 & 53,500 & 23,325 & MIAMI \\
\hline 0 & 0 & 0 & 1,205 & MICHIGAN \\
\hline 0 & 0 & 22,091 & 15,879 & MINNESOTA \\
\hline 0 & 0 & 0 & 0 & MONTREAL \\
\hline 59,858 & UA/NA & 149,404 & 9,654 & NEW MEXICO \\
\hline 15,706 & UA/NA & 115,461 & 123,790 & NEW YORK \\
\hline 23,739 & . & 48,544 & 80,485 & NORTH CAROLINA \\
\hline 6,583 & . & 76,687 & 13,628 & NORTHWESTERN \\
\hline 4,320 & 0 & 5,000 & 5,561 & OHIO STATE \\
\hline 26,543 & 45,987 & 83,009 & 42,635 & OKLAHOMA \\
\hline 2,916 & UA/NA & 10,263 & 1,009 & OKLAHOMA STATE \\
\hline
\end{tabular}




\section{Electronic Resources Expenditures}

One-time Electronic Resource Purchases
Ongoing Electronic Resource Purchases
Total

Electronic Resource Purchases
Total

Library Materials Expenditures
Electronic Resources as a \% of Library Materials Budget

(Survey Question Number)

(22)

(23)

$(22+23)$

(16)

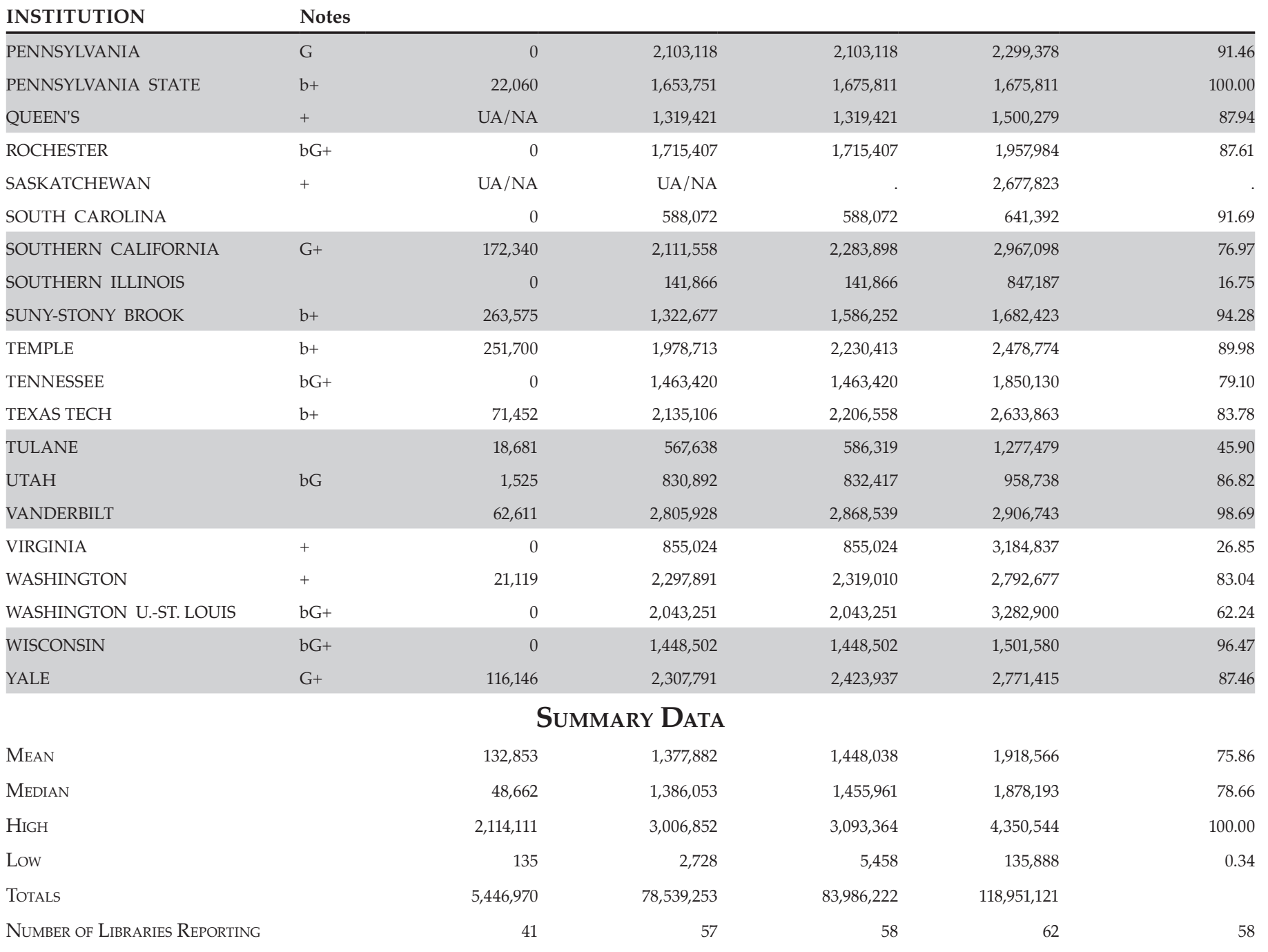

+- See footnotes

UA/NA - Unavailable or Not Applicable
G-Government documents not included in serials count $\mathrm{b}$ - Basis of volume count is bibliographic
- no data supplied 


\section{Electronic Resources ExPenditures}

Bibl. Utilities, Networks, etc. Bibl. Utilities, Networks, etc. Library Expenditures
Computer Hardware and Software Expenditures Document Delivery/
Interlibrary Loan Expenditures (24a)

(24b)
(25)
(26) (Survey Question Number)

\begin{tabular}{|c|c|c|c|c|}
\hline & & & & INSTITUTION \\
\hline $\mathrm{UA} / \mathrm{NA}$ & UA/NA & 6,888 & 128,970 & PENNSYLVANIA \\
\hline 0 & 0 & 21,413 & 0 & PENNSYLVANIA STATE \\
\hline UA/NA & UA/NA & UA/NA & UA/NA & QUEEN'S \\
\hline 2,643 & 0 & 126,220 & 33,973 & ROCHESTER \\
\hline UA/NA & UA/NA & $\mathrm{UA} / \mathrm{NA}$ & UA/NA & SASKATCHEWAN \\
\hline 5,222 & 0 & 40,590 & 2,464 & SOUTH CAROLINA \\
\hline 7,228 & 0 & 251,730 & 9,466 & SOUTHERN CALIFORNIA \\
\hline 0 & 0 & 28,646 & 4,484 & SOUTHERN ILLINOIS \\
\hline 26,475 & 330,118 & 340,869 & 9,563 & SUNY-STONY BROOK \\
\hline 5,147 & 0 & 268,931 & 3,641 & TEMPLE \\
\hline 12,883 & 1,703 & 55,791 & 7,495 & TENNESSEE \\
\hline 118,510 & 0 & 61,608 & 8,736 & TEXAS TECH \\
\hline 2,317 & 0 & 42,506 & 2,902 & TULANE \\
\hline 0 & 39,364 & 57,393 & 975 & UTAH \\
\hline 4,238 & 0 & 159,677 & 13,019 & VANDERBILT \\
\hline 7,415 & 0 & 102,150 & 27,810 & VIRGINIA \\
\hline . & . & 64,245 & 123,987 & WASHINGTON \\
\hline 0 & 811 & 49,146 & 1,946 & WASHINGTON U.-ST. LOUIS \\
\hline UA/NA & UA/NA & 30,160 & 205,637 & WISCONSIN \\
\hline 10,010 & & 81,250 & 26,931 & YALE \\
\hline \multicolumn{4}{|c|}{ SumMary Data } & \\
\hline 18,445 & 159,548 & 70,720 & 29,613 & MEAN \\
\hline 9,650 & 39,364 & 55,791 & 9,563 & Median \\
\hline 118,510 & 924,391 & 340,869 & 205,637 & HigH \\
\hline 965 & 421 & 123 & 717 & Low \\
\hline 664,032 & $1,435,936$ & $3,748,164$ & $1,510,255$ & TOTALS \\
\hline 36 & 9 & 53 & 51 & Number of Libraries Reporting \\
\hline
\end{tabular}




\section{Personnel and Public Services}

Notes

INSTITUTION

\begin{tabular}{|c|c|c|c|c|c|c|c|}
\hline ALABAMA & $\mathrm{bG}+$ & 3 & 3 & 0 & 6 & 2 & 63 \\
\hline ARIZONA & bG+ & 15 & 13 & 5 & 33 & 1 & 119 \\
\hline BOSTON & & 13 & 8 & 3 & 24 & 3 & 107 \\
\hline CALIFORNIA, DAVIS & $b+$ & 7 & 14 & 4 & 25 & 2 & 88 \\
\hline CALIFORNIA, IRVINE & bG & 8 & 15 & 5 & 28 & 7 & 128 \\
\hline CALIFORNIA, LOS ANGELES & bG+ & 15 & 11 & 14 & 40 & 1 & 80 \\
\hline CALIFORNIA, SAN DIEGO & $\mathrm{bG}+$ & 11 & 17 & 6 & 34 & UA/NA & UA/NA \\
\hline CASE WESTERN RESERVE & G+ & 7 & 10 & 8 & 25 & 2 & . \\
\hline CHICAGO & $\mathrm{G}+$ & 6 & 11 & 6 & 23 & 2 & 82 \\
\hline CINCINNATI & $\mathrm{b}+$ & 17 & 4 & 4 & 25 & 3 & 103 \\
\hline COLUMBIA & + & 14 & 11 & 1 & 26 & 3 & 95 \\
\hline CONNECTICUT & G+ & 14 & 13 & 3 & 30 & 3 & 94 \\
\hline CORNELL & + & 7 & 24 & 0 & 31 & 2 & 108 \\
\hline DARTMOUTH & bG+ + & 7 & 13 & 3 & 23 & 3 & 115 \\
\hline DUKE & $\mathrm{b}$ & 23 & 15 & 0 & 38 & 3 & 78 \\
\hline EMORY & + & 12 & 21 & 1 & 34 & 6 & 91 \\
\hline FLORIDA & + & 17 & 22 & 5 & 44 & 3 & 97 \\
\hline FLORIDA STATE & $\mathrm{b}+$ & 4 & 2 & 1 & 7 & 1 & 82 \\
\hline GEORGE WASHINGTON & bG+ & 14 & 17 & 6 & 37 & 4 & 113 \\
\hline GEORGETOWN & $\mathrm{b}+$ & 10 & 8 & 7 & 25 & 3 & 60 \\
\hline HARVARD & & 32 & 24 & 1 & 57 & . & . \\
\hline HAWAII & $b+$ & 3 & 4 & 0 & 7 & 1 & 78 \\
\hline HOWARD & bG & 7 & 11 & 5 & 23 & 3 & 113 \\
\hline ILLINOIS, CHICAGO & + & 16 & 28 & 8 & 52 & 5 & 102 \\
\hline INDIANA & $\mathrm{bG}+$ & 11 & 19 & 6 & 36 & 3 & 92 \\
\hline IOWA & $\mathrm{b}+$ & 11 & 11 & 10 & 32 & 3 & 100 \\
\hline KANSAS & + & 10 & 25 & 3 & 38 & 1 & 103 \\
\hline KENTUCKY & G+ & 14 & 13 & 7 & 34 & 3 & 110 \\
\hline LOUISIANA STATE & + & 1 & 6 & 1 & 8 & 1 & 88 \\
\hline MCGILL & $\mathrm{bG}+$ & 8 & 10 & 3 & 21 & 3 & 77 \\
\hline MCMASTER & $b+$ & 8 & 18 & 4 & 30 & 2 & 92 \\
\hline MIAMI & G+ & 13 & 23 & 0 & 36 & 5 & 103 \\
\hline MICHIGAN & bG+ & 15 & 17 & 13 & 45 & 2 & 97 \\
\hline MINNESOTA & $\mathrm{b}+$ & 15 & 27 & 13 & 55 & 4 & 96 \\
\hline MONTREAL & G+ & 10 & 22 & 0 & 32 & 12 & 93 \\
\hline NEW MEXICO & + & 16 & 22 & 2 & 40 & 2 & 95 \\
\hline NEW YORK & bG+ & 33 & 28 & 9 & 70 & 13 & 93 \\
\hline NORTH CAROLINA & G & 32 & 30 & 9 & 71 & 1 & 105 \\
\hline NORTHWESTERN & $\mathrm{G}+$ & 17 & 13 & 1 & 31 & 2 & 96 \\
\hline OHIO STATE & bG & 42 & 25 & 26 & 93 & 3 & 99 \\
\hline OKLAHOMA & + & 12 & 19 & 8 & 39 & 2 & 109 \\
\hline OKLAHOMA STATE & + & 4 & 6 & 2 & 12 & 2 & 104 \\
\hline
\end{tabular}

$24 \cdot$ ARL Academic Health Sciences Library Statistics 2008-2009
Professional Staff Support Staff Student Assistants (FTE)

(FTE)

(27a)

(27b)

Total Staff Staffed Library (FTE) Service Points Service Hours (Weekly)

(Survey Question Number)

(27c)

(27)

(28)

(29) 


\section{Personnel and Public Services}

Library Participants Reference Presentations in Group Transactions* to Groups* Presentations*
Initial

Circulation

Transactions
Total Total Items Circulation Loaned (ILL) Borrowed (ILL) Transactions
(30)

(31)

(32)

(33)

(34)

(35)

(36)

(Survey Question Number)

INSTITUTION

\begin{tabular}{|c|c|c|c|c|c|c|c|}
\hline 16 & 182 & 1,427 & 339 & 467 & 176 & 329 & ALABAMA \\
\hline 667 & 5,264 & 8,636 & 19,976 & 19,976 & 7,845 & 3,147 & ARIZONA \\
\hline 218 & 3,918 & 15,516 & 2,387 & 3,240 & 3,965 & 2,825 & BOSTON \\
\hline 327 & 1,727 & 19,564 & 54,575 & 71,643 & 22,241 & 3,674 & CALIFORNIA, DAVIS \\
\hline 84 & 2,394 & 7,513 & 18,430 & 41,193 & 3,154 & 3,382 & CALIFORNIA, IRVINE \\
\hline 179 & 3,697 & 9,691 & 27,949 & 83,961 & $\mathrm{UA} / \mathrm{NA}$ & UA/NA & CALIFORNIA, LOS ANGELES \\
\hline $\mathrm{UA} / \mathrm{NA}$ & UA/NA & UA/NA & UA/NA & UA/NA & $\mathrm{UA} / \mathrm{NA}$ & UA/NA & CALIFORNIA, SAN DIEGO \\
\hline 90 & 1,475 & UA/NA & 3,797 & 7,692 & 14,572 & 2,106 & CASE WESTERN RESERVE \\
\hline 47 & 707 & 3,216 & 20,217 & 21,668 & 9,294 & 1,707 & CHICAGO \\
\hline 250 & 2,672 & 6,500 & 9,527 & 12,184 & 8,059 & 2,538 & CINCINNATI \\
\hline 58 & 4,605 & 15,880 & 13,962 & 14,872 & 7,565 & 7,738 & COLUMBIA \\
\hline 188 & 1,500 & 11,982 & 10,841 & 13,777 & 9,018 & 941 & CONNECTICUT \\
\hline 203 & 1,206 & 7,323 & 15,227 & 19,429 & 2,559 & 3,843 & CORNELL \\
\hline 231 & 2,205 & UA/NA & 13,781 & 19,216 & 5,193 & 9,268 & DARTMOUTH \\
\hline 248 & 3,536 & 8,634 & 15,602 & 21,673 & 10,722 & 2,214 & DUKE \\
\hline 291 & 3,981 & 20,899 & 3,788 & 4,563 & 14,457 & 4,918 & EMORY \\
\hline 122 & 3,048 & 8,354 & 19,922 & 33,691 & 3,584 & 2,004 & FLORIDA \\
\hline 110 & 700 & 702 & 474 & 838 & 207 & 293 & FLORIDA STATE \\
\hline 463 & 6,987 & 12,039 & 9,121 & 20,114 & 1,242 & 595 & GEORGE WASHINGTON \\
\hline \multirow[t]{2}{*}{598} & 3,900 & 16,751 & 9,864 & & 1,149 & 1,009 & GEORGETOWN \\
\hline & & . & . & 30,838 & 4,451 & 1,142 & HARVARD \\
\hline 36 & 500 & 1,793 & 516 & 149 & 959 & 88 & HAWAII \\
\hline 73 & 2,974 & 10,000 & 15,581 & 20,825 & 1,127 & 836 & HOWARD \\
\hline 462 & 6,827 & 37,770 & 21,409 & 115,097 & 20,292 & 25,657 & ILLINOIS, CHICAGO \\
\hline 300 & 6,677 & 14,274 & 18,881 & 21,504 & 18,691 & 5,646 & INDIANA \\
\hline 279 & 6,124 & 5,495 & 11,279 & 12,366 & 21,044 & 12,346 & IOWA \\
\hline $\mathrm{UA} / \mathrm{NA}$ & UA/NA & UA/NA & UA/NA & 19,913 & 8,349 & 4,194 & KANSAS \\
\hline 308 & 2,868 & 10,414 & 12,462 & 17,019 & 7,800 & 9,893 & KENTUCKY \\
\hline 14 & 240 & UA/NA & 2,620 & UA/NA & 648 & 704 & LOUISIANA STATE \\
\hline 216 & 3,346 & 19,440 & 17,798 & 32,969 & 5,112 & 1,258 & MCGILL \\
\hline 203 & 1,996 & 11,632 & 23,007 & 29,724 & 6,470 & 5,131 & MCMASTER \\
\hline 703 & 2,064 & 6,944 & 6,931 & 9,567 & 13,437 & 1,814 & MIAMI \\
\hline 227 & 3,056 & 19,559 & 21,785 & 35,422 & 12,199 & 4,488 & MICHIGAN \\
\hline 378 & 8,094 & 16,395 & 23,466 & 36,679 & 41,715 & 6,694 & MINNESOTA \\
\hline 296 & 3,928 & 9,689 & 19,121 & 39,701 & 7,268 & 1,745 & MONTREAL \\
\hline 548 & 5,411 & 6,839 & 9,439 & 16,717 & 1,573 & 2,124 & NEW MEXICO \\
\hline 180 & 1,579 & 29,536 & 8,732 & 11,219 & 8,305 & 8,629 & NEW YORK \\
\hline 259 & 5,521 & 24,006 & 71,315 & 83,453 & 27,331 & 2,666 & NORTH CAROLINA \\
\hline 213 & 3,473 & 3,042 & 9,576 & 10,699 & 11,159 & 13,916 & NORTHWESTERN \\
\hline 143 & 3,362 & 7,766 & 11,197 & 33,558 & 17,733 & 4,421 & OHIO STATE \\
\hline 59 & 1,013 & 12,233 & 4,643 & 4,643 & 8,149 & 3,204 & OKLAHOMA \\
\hline 21 & 277 & 1,234 & 5,420 & 6,822 & 1,600 & 496 & OKLAHOMA STATE \\
\hline
\end{tabular}




\section{Personnel and Public Services}

Professional Staff Support Staff Student Assistants (FTE)

(FTE)

(FTE)

Total Staff

Staffed

Library

(FTE) Service Points Service Hours

(Weekly)

(Survey Question Number)

(27a) (27b)

(27c)

(27)

(28)

(29)

INSTITUTION

\begin{tabular}{|c|c|}
\hline PENNSYLVANIA & G \\
\hline PENNSYLVANIA STATE & $b+$ \\
\hline QUEEN'S & + \\
\hline ROCHESTER & bG+ \\
\hline SASKATCHEWAN & + \\
\hline \multicolumn{2}{|l|}{ SOUTH CAROLINA } \\
\hline SOUTHERN CALIFORNIA & G+ \\
\hline \multicolumn{2}{|l|}{ SOUTHERN ILLINOIS } \\
\hline SUNY-STONY BROOK & $\mathrm{b}$ \\
\hline TEMPLE & $b+$ \\
\hline TENNESSEE & bG+ \\
\hline TEXAS TECH & $b+$ \\
\hline \multicolumn{2}{|l|}{ TULANE } \\
\hline UTAH & $\mathrm{bG}$ \\
\hline \multicolumn{2}{|l|}{ VANDERBILT } \\
\hline VIRGINIA & + \\
\hline WASHINGTON & + \\
\hline WASHINGTON U.-ST. LOUIS & bG+ \\
\hline WISCONSIN & bG+ + \\
\hline YALE & G+ \\
\hline
\end{tabular}

$\begin{array}{rr}10 & 12 \\ 6 & 9 \\ 7 & 11 \\ 21 & 8\end{array}$

\begin{tabular}{rrrr}
7 & 29 & 2 & 100 \\
3 & 18 & 3 & 106 \\
2 & 20 & 2 & 97 \\
3 & 32 & 4 & 106 \\
2 & 16 & 2 & 89 \\
2 & 18 & 2 & 88 \\
7 & 45 & 5 & 102 \\
1 & 22 & 3 & 90 \\
10 & 38 & 3 & 100 \\
6 & 31 & 5 & 108 \\
5 & 42 & 4 & $\mathrm{UA} / \mathrm{NA}$ \\
17 & 68 & 15 & 105 \\
5 & 18 & 1 & 93 \\
14 & 55 & 4 & 100 \\
1 & 45 & 2 & 111 \\
\hline 0 & 35 & 3 & 100 \\
9 & 56 & 3 & 80 \\
1 & 43 & 2 & 102 \\
11 & 41 & 2 & 96 \\
2 & 39 & 2 & 107
\end{tabular}

\section{Summary Data}

MEAN

MEdian

$\mathrm{HigH}$

Low

TOTALS

NuMber OF Libraries REPORTING

+- See footnotes

UA/NA - Unavailable or Not Applicable

$\begin{array}{rr}14 & 15 \\ 13 & 15 \\ 42 & 30 \\ 1 & 2 \\ 856 & 954 \\ 62 & 62\end{array}$

G-Government documents not included in serials count

b-Basis of volume count is bibliographic

$\begin{array}{rrrr}6 & 34 & 3 & 97 \\ 5 & 33 & 3 & 98 \\ 26 & 93 & 15 & 128 \\ 1 & 6 & 1 & 60 \\ 321 & 2,131 & 196 & 5,628 \\ 55 & 62 & 60 & 58\end{array}$




\section{Personnel and Public Services}

Library Participants Reference Presentations in Group Transactions* to Groups* Presentations*
Initial

Circulation

Transactions
Total Total Items Circulation Loaned (ILL) Borrowed (ILL) Transactions

(30)

(31)

(32)

(33)

(34)

(35)

(36)

(Survey Question Number)

INSTITUTION

\begin{tabular}{|c|c|c|c|c|c|c|c|}
\hline 156 & 2,911 & UA/NA & 7,138 & 33,654 & 7,396 & 23,130 & PENNSYLVANIA \\
\hline 118 & 1,486 & 10,772 & 10,582 & 11,292 & 8,999 & 1,341 & PENNSYLVANIA STATE \\
\hline 363 & 3,005 & 5,375 & 8,284 & 9,011 & 5,082 & 1,834 & QUEEN'S \\
\hline 151 & 1,491 & 7,463 & 4,578 & 6,034 & 4,407 & 4,190 & ROCHESTER \\
\hline 195 & 3,285 & 3,115 & 15,000 & 22,742 & 3,644 & 3,063 & SASKATCHEWAN \\
\hline 51 & 973 & 1,037 & 1,439 & 2,125 & 1,648 & 652 & SOUTH CAROLINA \\
\hline 113 & 2,769 & 7,759 & 21,852 & 28,476 & 12,579 & 1,142 & SOUTHERN CALIFORNIA \\
\hline 142 & 838 & 3,827 & 30,760 & 36,188 & 7,304 & 2,230 & SOUTHERN ILLINOIS \\
\hline 173 & 2,298 & 15,036 & 4,823 & 7,273 & 6,300 & 792 & SUNY-STONY BROOK \\
\hline 42 & 701 & 2,273 & 5,278 & 7,291 & 1,034 & 1,246 & TEMPLE \\
\hline 288 & 3,951 & 17,992 & 2,183 & 3,561 & 5,236 & 2,215 & TENNESSEE \\
\hline 495 & 4,674 & 17,065 & 29,402 & 75,179 & 5,315 & 2,501 & TEXAS TECH \\
\hline 74 & 762 & 10,848 & 7,227 & 8,527 & 3,307 & 3,955 & TULANE \\
\hline 272 & 2,781 & 48,287 & 34,228 & 35,974 & 9,227 & 10,209 & UTAH \\
\hline 277 & 5,994 & 4,451 & 11,203 & 12,238 & 8,225 & 3,806 & VANDERBILT \\
\hline 475 & 3,224 & 8,105 & 8,692 & 11,170 & 5,590 & 2,859 & VIRGINIA \\
\hline 261 & 2,772 & 11,178 & 19,256 & 56,649 & 12,871 & 5,603 & WASHINGTON \\
\hline 323 & 3,165 & 10,601 & 13,788 & 16,182 & 22,789 & 2,315 & WASHINGTON U.-ST. LOUIS \\
\hline 237 & 4,145 & 3,484 & 10,535 & 15,525 & 11,732 & 23,467 & WISCONSIN \\
\hline 293 & 4,203 & 8,441 & UA/NA & 21,487 & 12,309 & 10,554 & YALE \\
\hline \multicolumn{8}{|c|}{ SumMary Data } \\
\hline 234 & 3,025 & 11,269 & 14,331 & 24,062 & 8,757 & 4,612 & MEAN \\
\hline 216 & 2,974 & 9,689 & 11,200 & 19,216 & 7,481 & 2,746 & MEdian \\
\hline 703 & 8,094 & 48,287 & 71,315 & 115,097 & 41,715 & 25,657 & HigH \\
\hline 14 & 182 & 702 & 339 & 149 & 176 & 88 & Low \\
\hline 13,807 & 178,462 & 619,797 & 831,205 & $1,419,659$ & 525,408 & 276,727 & TOTALS \\
\hline 59 & 59 & 55 & 58 & 59 & 60 & 60 & Number of Libraries Reporting \\
\hline
\end{tabular}

\footnotetext{
${ }^{*}$ Figures in italics are derived from a sampling method rather than an actual annual count.
} 
Rank Order Table 1: Volumes in Library

\begin{tabular}{|c|c|c|c|c|c|c|c|c|c|}
\hline & INSTITUTION & $\begin{array}{l}\text { HS LiBRARY } \\
\text { VOLUMES }\end{array}$ & $\begin{array}{r}\text { Total } \\
\text { VoLUMES }\end{array}$ & $\begin{array}{c}\text { HS } \% \text { of } \\
\text { TOTAL }\end{array}$ & & INSTITUTION & $\begin{array}{l}\text { HS LIBRARY } \\
\text { VOLUMES }\end{array}$ & $\begin{array}{r}\text { Total } \\
\text { Volumes }\end{array}$ & $\begin{array}{c}\text { HS } \% \text { OF } \\
\text { TOTAL }\end{array}$ \\
\hline 1 & CHICAGO & 854,643 & $8,830,151$ & 9.68 & 32 & CALIFORNIA, SAN DIEGO & 229,450 & $3,651,393$ & 6.28 \\
\hline & ILLINOIS, CHICAGO & 748,567 & $2,515,007$ & 29.76 & 33 & SOUTHERN CALIFORNIA & 224,275 & $4,124,253$ & 5.44 \\
\hline 3 & HARVARD & 695,415 & $16,557,002$ & 4.20 & 34 & ARIZONA & 222,477 & $5,794,299$ & 3.84 \\
\hline 4 & CALIFORNIA, LOS ANGELES & 670,751 & $9,045,818$ & 7.42 & 35 & TENNESSEE & 221,223 & $3,322,418$ & 6.66 \\
\hline 5 & MICHIGAN & 633,442 & $9,575,256$ & 6.62 & 36 & MIAMI & 207,845 & $3,300,370$ & 6.30 \\
\hline 6 & MINNESOTA & 551,030 & $6,975,576$ & 7.90 & 37 & INDIANA & 207,693 & $8,543,025$ & 2.43 \\
\hline 7 & YALE & 488,300 & $12,564,157$ & 3.89 & 38 & PENNSYLVANIA & 204,395 & $6,223,214$ & 3.28 \\
\hline 8 & MCGILL & 485,277 & $4,128,321$ & 11.75 & 39 & CORNELL & 201,919 & $8,036,029$ & 2.51 \\
\hline 9 & CASE WESTERN RESERVE & 423,707 & $2,777,529$ & 15.25 & 40 & CONNECTICUT & 198,485 & $3,982,991$ & 4.98 \\
\hline 10 & CALIFORNIA, IRVINE & 377,512 & $3,145,926$ & 12.00 & 41 & UTAH & 197,641 & $3,418,976$ & 5.78 \\
\hline 11 & CALIFORNIA, DAVIS & 374,218 & $4,156,170$ & 9.00 & 42 & VANDERBILT & 195,901 & $3,467,542$ & 5.65 \\
\hline 12 & IOWA & 359,484 & $5,155,258$ & 6.97 & 43 & SASKATCHEWAN & 191,013 & $2,361,589$ & 8.09 \\
\hline 13 & WASHINGTON & 353,626 & $7,549,765$ & 4.68 & 44 & KANSAS & 175,370 & $4,271,113$ & 4.11 \\
\hline 14 & NORTH CAROLINA & 341,582 & $6,735,325$ & 5.07 & 45 & SOUTHERN ILLINOIS & 168,406 & $3,149,701$ & 5.35 \\
\hline 15 & COLUMBIA & 338,436 & $10,449,223$ & 3.24 & 46 & TULANE & 158,988 & $4,004,458$ & 3.97 \\
\hline 16 & MONTREAL & 335,595 & $3,180,763$ & 10.55 & 47 & NEW MEXICO & 154,661 & $3,117,590$ & 4.96 \\
\hline 17 & OKLAHOMA & 329,056 & $5,433,036$ & 6.06 & 48 & BOSTON & 145,148 & $2,864,562$ & 5.07 \\
\hline 18 & FLORIDA & 319,367 & $4,299,252$ & 7.43 & 49 & TEMPLE & 145,124 & $3,761,933$ & 3.86 \\
\hline 19 & WISCONSIN & 318,124 & $8,310,732$ & 3.83 & 50 & MCMASTER & 144,737 & $2,014,067$ & 7.19 \\
\hline 20 & TEXAS TECH & 314,234 & $2,675,872$ & 11.74 & 51 & QUEEN'S & 142,409 & $2,577,143$ & 5.53 \\
\hline 21 & WASHINGTON U.-ST. LOUIS & 298,938 & $4,281,213$ & 6.98 & 52 & HOWARD & 135,021 & $2,589,888$ & 5.21 \\
\hline 22 & CINCINNATI & 293,192 & $3,715,957$ & 7.89 & 53 & VIRGINIA & 124,925 & $5,605,891$ & 2.23 \\
\hline 23 & NORTHWESTERN & 292,465 & $4,930,613$ & 5.93 & 54 & SOUTH CAROLINA & 117,118 & $3,675,054$ & 3.19 \\
\hline 24 & OHIO STATE & 288,002 & $6,206,443$ & 4.64 & 55 & GEORGE WASHINGTON & 98,900 & $2,268,571$ & 4.36 \\
\hline 25 & SUNY-STONY BROOK & 274,334 & $2,277,714$ & 12.04 & 56 & GEORGETOWN & 98,598 & $3,431,948$ & 2.87 \\
\hline 26 & ROCHESTER & 270,949 & $3,740,714$ & 7.24 & 57 & OKLAHOMA STATE & 60,392 & $2,932,910$ & 2.06 \\
\hline 27 & DARTMOUTH & 260,542 & $2,848,521$ & 9.15 & 58 & LOUISIANA STATE & 49,090 & $4,112,774$ & 1.19 \\
\hline 28 & DUKE & 247,664 & $6,031,761$ & 4.11 & 59 & PENNSYLVANIA STATE & 48,493 & $5,365,489$ & 0.90 \\
\hline 29 & KENTUCKY & 243,486 & $3,784,382$ & 6.43 & 60 & HAWAII & 33,766 & $3,588,005$ & 0.94 \\
\hline 30 & EMORY & 241,736 & $3,479,536$ & 6.95 & 61 & ALABAMA & 21,322 & $3,396,810$ & 0.63 \\
\hline 31 & NEW YORK & 230,572 & $5,191,617$ & 4.44 & 62 & FLORIDA STATE & 12,344 & $3,034,491$ & 0.41 \\
\hline
\end{tabular}




\section{Rank Order Table 2: Volumes Added (Gross)}

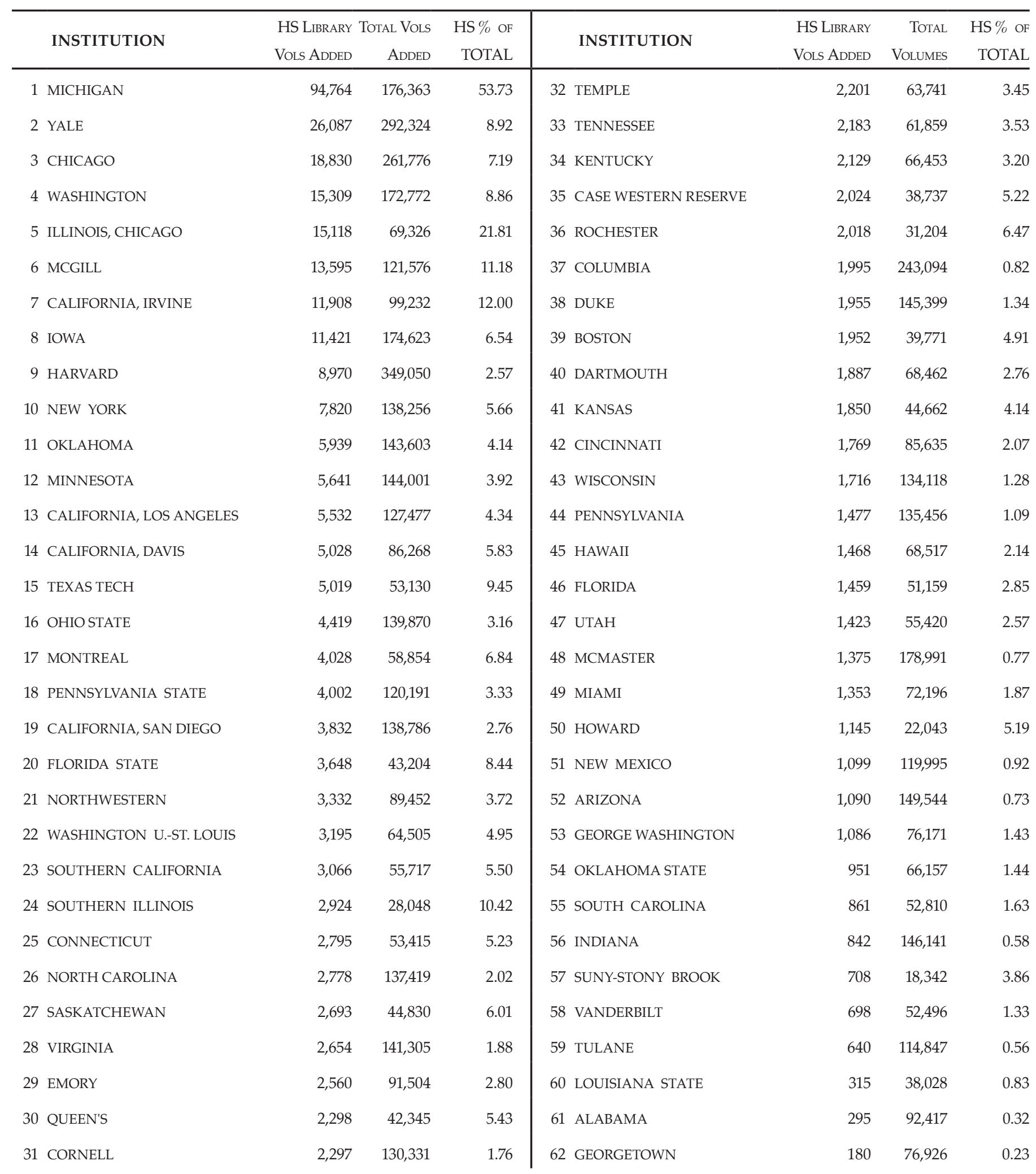


Rank Order Table 3: Current Serials (Total)

\begin{tabular}{|c|c|c|c|c|c|c|c|c|}
\hline INSTITUTION & $\begin{array}{r}\text { HS LIBRARY } \\
\text { SERIALS }\end{array}$ & $\begin{array}{r}\text { Total } \\
\text { Serials }\end{array}$ & $\begin{array}{r}\mathrm{HS} \% \text { OF } \\
\text { TOTAL }\end{array}$ & & INSTITUTION & $\begin{array}{r}\text { HS LIBRARY } \\
\text { SERIALS }\end{array}$ & $\begin{array}{r}\text { Total } \\
\text { Serials }\end{array}$ & $\begin{array}{r}\text { HS \% OF } \\
\text { TOTAL }\end{array}$ \\
\hline 1 MICHIGAN & 30,971 & 70,047 & 44.21 & 32 & MONTREAL & 3,836 & 90,160 & 4.25 \\
\hline 2 SOUTH CAROLINA & 30,485 & 66,309 & 45.97 & 33 & UTAH & 3,497 & 71,926 & 4.86 \\
\hline 3 CHICAGO & 29,290 & 102,767 & 28.50 & 34 & LOUISIANA STATE & 3,212 & 104,177 & 3.08 \\
\hline 4 TEXAS TECH & 22,483 & 84,317 & 26.66 & 35 & DARTMOUTH & 3,136 & 64,745 & 4.84 \\
\hline 5 CONNECTICUT & 16,586 & 92,884 & 17.86 & 36 & ALABAMA & 2,816 & 81,462 & 3.46 \\
\hline 6 ARIZONA & 10,785 & 84,367 & 12.78 & 37 & SOUTHERN CALIFORNIA & 2,749 & 98,728 & 2.78 \\
\hline 7 YALE & 10,407 & 96,721 & 10.76 & 38 & GEORGE WASHINGTON & 2,136 & 74,329 & 2.87 \\
\hline 8 MIAMI & 9,910 & 76,869 & 12.89 & 39 & NEW MEXICO & 2,039 & 77,094 & 2.64 \\
\hline 9 NORTHWESTERN & 9,644 & 98,844 & 9.76 & 40 & TENNESSEE & 1,787 & 58,377 & 3.06 \\
\hline 10 CORNELL & 9,500 & 102,000 & 9.31 & 41 & CALIFORNIA, DAVIS & 1,343 & 73,912 & 1.82 \\
\hline 11 EMORY & 9,087 & 83,514 & 10.88 & 42 & ILLINOIS, CHICAGO & 1,238 & 67,318 & 1.84 \\
\hline 12 TEMPLE & 7,598 & 62,173 & 12.22 & 43 & SOUTHERN ILLINOIS & 1,152 & 51,350 & 2.24 \\
\hline 13 PENNSYLVANIA STATE & 7,262 & 99,091 & 7.33 & 44 & KANSAS & 920 & 73,613 & 1.25 \\
\hline 14 INDIANA & 7,105 & 84,933 & 8.37 & 45 & FLORIDA STATE & 698 & 78,295 & 0.89 \\
\hline 15 MCGILL & 6,792 & 65,536 & 10.36 & 46 & MCMASTER & 293 & 69,781 & 0.42 \\
\hline 16 HOWARD & 6,688 & 24,307 & 27.51 & 47 & KENTUCKY & 222 & 78,194 & 0.28 \\
\hline 17 BOSTON & 6,508 & 57,755 & 11.27 & 48 & FLORIDA & 211 & 89,741 & 0.24 \\
\hline 18 CALIFORNIA, IRVINE & 6,466 & 53,891 & 12.00 & 49 & ROCHESTER & 124 & 36,033 & 0.34 \\
\hline 19 OHIO STATE & 6,411 & 90,156 & 7.11 & 50 & CASE WESTERN RESERVE & 91 & 75,083 & 0.12 \\
\hline 20 IOWA & 5,788 & 72,271 & 8.01 & 51 & WASHINGTON & 0 & 63,575 & 0.00 \\
\hline 21 SASKATCHEWAN & 5,530 & 40,981 & 13.49 & 52 & CALIFORNIA, LOS ANGELES & $\mathrm{UA} / \mathrm{NA}$ & 38,975 & $\mathrm{UA} / \mathrm{NA}$ \\
\hline 22 DUKE & 5,108 & 91,931 & 5.56 & 52 & CALIFORNIA, SAN DIEGO & $\mathrm{UA} / \mathrm{NA}$ & 84,513 & $\mathrm{UA} / \mathrm{NA}$ \\
\hline 23 PENNSYLVANIA & 4,922 & 72,688 & 6.77 & 52 & CINCINNATI & $\mathrm{UA} / \mathrm{NA}$ & 103,066 & $\mathrm{UA} / \mathrm{NA}$ \\
\hline 24 WISCONSIN & 4,740 & 101,596 & 4.67 & 52 & COLUMBIA & $\mathrm{UA} / \mathrm{NA}$ & 144,787 & $\mathrm{UA} / \mathrm{NA}$ \\
\hline 25 HAWAII & 4,688 & 67,863 & 6.91 & 52 & HARVARD & $\mathrm{UA} / \mathrm{NA}$ & 132,107 & $\mathrm{UA} / \mathrm{NA}$ \\
\hline 26 SUNY-STONY BROOK & 4,483 & 95,517 & 4.69 & 52 & MINNESOTA & $\mathrm{UA} / \mathrm{NA}$ & 90,860 & $\mathrm{UA} / \mathrm{NA}$ \\
\hline 27 VANDERBILT & 4,410 & 55,260 & 7.98 & 52 & NEW YORK & $\mathrm{UA} / \mathrm{NA}$ & 108,454 & $\mathrm{UA} / \mathrm{NA}$ \\
\hline 28 GEORGETOWN & 4,153 & 70,518 & 5.89 & 52 & OKLAHOMA & UA/NA & 69,621 & $\mathrm{UA} / \mathrm{NA}$ \\
\hline 29 NORTH CAROLINA & 4,096 & 80,132 & 5.11 & 52 & OKLAHOMA STATE & UA/NA & 70,803 & UA/NA \\
\hline 30 WASHINGTON U.-ST. LOUIS & 4,012 & 71,905 & 5.58 & 52 & QUEEN'S & UA/NA & 69,981 & $\mathrm{UA} / \mathrm{NA}$ \\
\hline 31 TULANE & 4,000 & 83,452 & 4.79 & 52 & VIRGINIA & UA/NA & 117,749 & $\mathrm{UA} / \mathrm{NA}$ \\
\hline
\end{tabular}


Rank Order Table 4: Total Library Expenditures

\begin{tabular}{|c|c|c|c|c|c|c|c|c|}
\hline INSTITUTION & $\begin{array}{c}\text { HS LIBRARY } \\
\text { EXPENDITURES }\end{array}$ & $\begin{array}{r}\text { TOTAL } \\
\text { EXPENDITURES }\end{array}$ & $\begin{array}{r}\mathrm{HS} \% \text { OF } \\
\text { TOTAL }\end{array}$ & & INSTITUTION & $\begin{array}{l}\text { HS LIBRARY } \\
\text { EXPENDITURES }\end{array}$ & $\begin{array}{r}\text { TOTAL } \\
\text { EXPENDITURES }\end{array}$ & $\begin{array}{r}\text { HS \% OF } \\
\text { TOTAL }\end{array}$ \\
\hline 1 WASHINGTON & $8,140,690$ & $43,956,131$ & 18.52 & 32 & 2 SUNY-STONY BROOK & $3,791,832$ & $13,772,703$ & 27.53 \\
\hline 2 NORTH CAROLINA & $7,770,531$ & $40,769,990$ & 19.06 & 33 & 3 MONTREAL & $3,782,830$ & $29,812,219$ & 12.69 \\
\hline 3 HARVARD & $6,533,719$ & $122,980,135$ & 5.31 & 34 & 4 PENNSYLVANIA & $3,762,083$ & $39,587,076$ & 9.50 \\
\hline 4 MINNESOTA & $6,239,579$ & $41,225,580$ & 15.14 & 35 & 5 CALIFORNIA, SAN DIEGO & $3,665,036$ & $30,076,158$ & 12.19 \\
\hline 5 WASHINGTON U.-ST. LOUIS & $6,143,409$ & $28,230,503$ & 21.76 & 36 & 6 CORNELL & $3,563,377$ & $46,546,720$ & 7.66 \\
\hline 6 NEW YORK & $6,003,589$ & $45,893,186$ & 13.08 & 37 & 7 TENNESSEE & $3,487,829$ & $23,515,580$ & 14.83 \\
\hline 7 VANDERBILT & $5,889,596$ & $24,395,492$ & 24.14 & 38 & 3 SASKATCHEWAN & $3,430,158$ & $18,235,214$ & 18.81 \\
\hline 8 SOUTHERN CALIFORNIA & $5,740,036$ & $38,993,308$ & 14.72 & 39 & CINCINNATI & $3,391,477$ & $20,330,143$ & 16.68 \\
\hline 9 OHIO STATE & $5,685,776$ & $38,818,365$ & 14.65 & 40 & KANSAS & $3,377,400$ & $23,042,922$ & 14.66 \\
\hline 10 YALE & $5,523,740$ & $88,942,761$ & 6.21 & 41 & KENTUCKY & $3,350,411$ & $21,734,374$ & 15.42 \\
\hline 11 CHICAGO & $5,343,542$ & $35,646,147$ & 14.99 & 42 & NEW MEXICO & $3,349,138$ & $20,809,647$ & 16.09 \\
\hline 12 VIRGINIA & $5,286,780$ & $34,248,073$ & 15.44 & 43 & 3 GEORGETOWN & $3,092,083$ & $28,040,057$ & 11.03 \\
\hline 13 TEXAS TECH & $5,053,806$ & $26,049,443$ & 19.40 & 44 & 4 CASE WESTERN RESERVE & $2,943,992$ & $14,019,260$ & 21.00 \\
\hline 14 CALIFORNIA, LOS ANGELES & $4,967,481$ & $50,205,645$ & 9.89 & 45 & 5 MCGILL & $2,797,751$ & $28,349,450$ & 9.87 \\
\hline 15 ILLINOIS, CHICAGO & $4,954,663$ & $20,616,384$ & 24.03 & 46 & 5 BOSTON & $2,750,466$ & $21,486,285$ & 12.80 \\
\hline 16 EMORY & $4,817,241$ & $34,720,327$ & 13.87 & 47 & 7 QUEEN'S & $2,672,638$ & $16,939,189$ & 15.78 \\
\hline 17 FLORIDA & $4,692,615$ & $28,147,202$ & 16.67 & 48 & CALIFORNIA, DAVIS & $2,655,911$ & $19,932,904$ & 13.32 \\
\hline 18 INDIANA & $4,636,115$ & $33,147,603$ & 13.99 & 49 & MCMASTER & $2,645,078$ & $17,449,235$ & 15.16 \\
\hline 19 MIAMI & $4,344,461$ & $26,584,581$ & 16.34 & 50 & CALIFORNIA, IRVINE & $2,633,748$ & $21,947,912$ & 12.00 \\
\hline 20 TEMPLE & $4,193,993$ & $22,861,433$ & 18.35 & 51 & 1 PENNSYLVANIA STATE & $2,590,921$ & $49,451,130$ & 5.24 \\
\hline 21 NORTHWESTERN & $4,143,803$ & $30,270,763$ & 13.69 & 52 & 2 IOWA & $2,568,927$ & $29,517,181$ & 8.70 \\
\hline 22 OKLAHOMA & $4,095,123$ & $23,865,613$ & 17.16 & 53 & 3 DARTMOUTH & $2,422,847$ & $19,702,562$ & 12.30 \\
\hline 23 CONNECTICUT & $4,092,075$ & $26,831,009$ & 15.25 & 54 & 4 HOWARD & $2,117,244$ & $9,347,005$ & 22.65 \\
\hline 24 UTAH & $4,026,695$ & $23,948,377$ & 16.81 & 55 & 5 TULANE & $1,900,678$ & $14,875,907$ & 12.78 \\
\hline 25 DUKE & $4,026,532$ & $38,013,641$ & 10.59 & 56 & 6 SOUTHERN ILLINOIS & $1,839,339$ & $15,228,311$ & 12.08 \\
\hline 26 WISCONSIN & $3,966,019$ & $43,732,756$ & 9.07 & 57 & 7 SOUTH CAROLINA & $1,398,540$ & $19,284,992$ & 7.25 \\
\hline 27 GEORGE WASHINGTON & $3,950,880$ & $26,491,902$ & 14.91 & 58 & 3 FLORIDA STATE & $1,224,938$ & $15,750,486$ & 7.78 \\
\hline 28 ROCHESTER & $3,856,755$ & $18,887,593$ & 20.42 & 59 & OKLAHOMA STATE & $1,219,764$ & $17,886,909$ & 6.82 \\
\hline 29 COLUMBIA & $3,847,309$ & $63,320,237$ & 6.08 & 60 & HAWAII & 827,284 & $19,076,939$ & 4.34 \\
\hline 30 MICHIGAN & $3,824,455$ & $53,134,323$ & 7.20 & 61 & 1 LOUISIANA STATE & 619,349 & $13,428,011$ & 4.61 \\
\hline 31 ARIZONA & $3,797,023$ & $30,645,753$ & 12.39 & 62 & 2 ALABAMA & 359,925 & $17,430,914$ & 2.06 \\
\hline
\end{tabular}


Rank Order Table 5: Total StafF

\begin{tabular}{|c|c|c|c|c|c|c|c|c|}
\hline INSTITUTION & \multicolumn{2}{|c|}{$\begin{array}{l}\text { HS LibRARY TOTAL STAFF } \\
\text { StaFF }\end{array}$} & $\begin{array}{r}\mathrm{HS} \% \text { OF } \\
\text { TOTAL } \\
\end{array}$ & \multicolumn{2}{|r|}{ INSTITUTION } & \multicolumn{2}{|c|}{$\begin{array}{l}\text { HS LibRARYTOTAL STAFF } \\
\text { StaFF }\end{array}$} & $\begin{array}{r}\mathrm{HS} \% \text { OF } \\
\text { TOTAL } \\
\end{array}$ \\
\hline 1 OHIO STATE & 93 & 469 & 19.83 & 32 & IOWA & 32 & 282 & 11.35 \\
\hline 2 NORTH CAROLINA & 71 & 468 & 15.17 & 32 & MONTREAL & 32 & 365 & 8.77 \\
\hline 3 NEW YORK & 70 & 471 & 14.86 & 32 & ROCHESTER & 32 & 215 & 14.88 \\
\hline 4 TEXAS TECH & 68 & 337 & 20.18 & 35 & CORNELL & 31 & 524 & 5.92 \\
\hline 5 HARVARD & 57 & 1,214 & 4.70 & 35 & NORTHWESTERN & 31 & 343 & 9.04 \\
\hline 6 WASHINGTON & 56 & 521 & 10.75 & 35 & TEMPLE & 31 & 213 & 14.55 \\
\hline 7 MINNESOTA & 55 & 391 & 14.07 & 38 & CONNECTICUT & 30 & 198 & 15.15 \\
\hline 7 UTAH & 55 & 323 & 17.03 & 38 & MCMASTER & 30 & 168 & 17.86 \\
\hline 9 ILLINOIS, CHICAGO & 52 & 185 & 28.11 & 40 & PENNSYLVANIA & 29 & 405 & 7.16 \\
\hline 10 MICHIGAN & 45 & 584 & 7.71 & 41 & CALIFORNIA, IRVINE & 28 & 230 & 12.17 \\
\hline 10 SOUTHERN CALIFORNIA & 45 & 373 & 12.06 & 42 & COLUMBIA & 26 & 657 & 3.96 \\
\hline 10 VANDERBILT & 45 & 244 & 18.44 & 43 & CALIFORNIA, DAVIS & 25 & 208 & 12.02 \\
\hline 13 FLORIDA & 44 & 487 & 9.03 & 43 & CASE WESTERN RESERVE & 25 & 134 & 18.66 \\
\hline 14 WASHINGTON U.-ST. LOUIS & 43 & 258 & 16.67 & 43 & CINCINNATI & 25 & 176 & 14.20 \\
\hline 15 TENNESSEE & 42 & 249 & 16.87 & 43 & GEORGETOWN & 25 & 261 & 9.58 \\
\hline 16 WISCONSIN & 41 & 607 & 6.75 & 47 & BOSTON & 24 & 281 & 8.54 \\
\hline 17 CALIFORNIA, LOS ANGELES & 40 & 580 & 6.90 & 48 & CHICAGO & 23 & 314 & 7.32 \\
\hline 17 NEW MEXICO & 40 & 235 & 17.02 & 48 & DARTMOUTH & 23 & 196 & 11.73 \\
\hline 19 OKLAHOMA & 39 & 233 & 16.74 & 48 & HOWARD & 23 & 157 & 14.65 \\
\hline 19 YALE & 39 & 716 & 5.45 & 51 & SOUTHERN ILLINOIS & 22 & 191 & 11.52 \\
\hline 21 DUKE & 38 & 348 & 10.92 & 52 & MCGILL & 21 & 250 & 8.40 \\
\hline 21 KANSAS & 38 & 291 & 13.06 & 53 & QUEEN'S & 20 & 170 & 11.76 \\
\hline 21 SUNY-STONY BROOK & 38 & 144 & 26.39 & 54 & PENNSYLVANIA STATE & 18 & 596 & 3.02 \\
\hline 24 GEORGE WASHINGTON & 37 & 245 & 15.10 & 54 & SOUTH CAROLINA & 18 & 274 & 6.57 \\
\hline 25 INDIANA & 36 & 415 & 8.67 & 54 & TULANE & 18 & 153 & 11.76 \\
\hline 25 MIAMI & 36 & 276 & 13.04 & 57 & SASKATCHEWAN & 16 & 154 & 10.39 \\
\hline 27 VIRGINIA & 35 & 378 & 9.26 & 58 & OKLAHOMA STATE & 12 & 217 & 5.53 \\
\hline 28 CALIFORNIA, SAN DIEGO & 34 & 361 & 9.42 & 59 & LOUISIANA STATE & 8 & 187 & 4.28 \\
\hline 28 EMORY & 34 & 283 & 12.01 & 60 & FLORIDA STATE & 7 & 224 & 3.13 \\
\hline 28 KENTUCKY & 34 & 268 & 12.69 & 60 & HAWAII & 7 & 238 & 2.94 \\
\hline 31 ARIZONA & 33 & 287 & 11.50 & 62 & ALABAMA & 6 & 203 & 2.96 \\
\hline
\end{tabular}


Rank Order Table 6: Total Electronic Resources Expenditures

\begin{tabular}{|c|c|c|c|c|c|c|c|c|}
\hline INSTITUTION & $\begin{array}{l}\text { HS LIBRARY } \\
\text { E-RESOURCES }\end{array}$ & $\begin{array}{r}\text { Total } \\
\text { E-RESOURCES }\end{array}$ & $\begin{array}{r}\mathrm{HS} \% \text { OF } \\
\text { TOTAL }\end{array}$ & & INSTITUTION & $\begin{array}{l}\text { HS LIBRARY } \\
\text { E-RESOURCES }\end{array}$ & $\begin{array}{r}\text { Total } \\
\text { E-Resources }\end{array}$ & $\begin{array}{r}\text { HS \% OF } \\
\text { TOTAL }\end{array}$ \\
\hline 1 CHICAGO & $3,093,364$ & $7,030,483$ & 44.00 & 32 & QUEEN'S & $1,319,421$ & $4,960,207$ & 26.60 \\
\hline 2 NORTH CAROLINA & $3,077,248$ & $8,565,526$ & 35.93 & 33 & 3 ARIZONA & $1,308,613$ & $8,696,274$ & 15.05 \\
\hline 3 VANDERBILT & $2,868,539$ & $7,932,073$ & 36.16 & 34 & CINCINNATI & $1,284,455$ & $5,554,516$ & 23.12 \\
\hline 4 INDIANA & $2,726,807$ & $7,561,199$ & 36.06 & 35 & 5 DUKE & $1,216,650$ & $8,384,850$ & 14.5 \\
\hline 5 YALE & $2,423,937$ & $9,418,024$ & 25.74 & 36 & KANSAS & $1,177,031$ & $5,973,543$ & 19.7 \\
\hline 6 MINNESOTA & $2,391,536$ & $9,327,938$ & 25.64 & 37 & 7 NEW MEXICO & $1,137,989$ & $4,203,707$ & 27.07 \\
\hline 7 WASHINGTON & $2,319,010$ & $8,672,713$ & 26.74 & 38 & 3 CALIFORNIA, DAVIS & $1,131,891$ & $4,888,620$ & 23.15 \\
\hline 8 SOUTHERN CALIFORNIA & $2,283,898$ & $6,676,513$ & 34.21 & 39 & EMORY & $1,103,744$ & $6,117,121$ & 18.04 \\
\hline 9 NEW YORK & $2,253,833$ & $9,874,054$ & 22.83 & 40 & CORNELL & $1,083,871$ & $7,605,859$ & 14.25 \\
\hline 10 TEMPLE & $2,230,413$ & $7,800,437$ & 28.59 & 41 & MCGILL & $1,076,907$ & $7,667,117$ & 14.05 \\
\hline 11 TEXAS TECH & $2,206,558$ & $5,854,021$ & 37.69 & 42 & HOWARD & $1,066,473$ & $2,547,396$ & 41.87 \\
\hline 12 NORTHWESTERN & $2,156,732$ & $7,838,852$ & 27.51 & 43 & DARTMOUTH & $1,032,675$ & $6,289,015$ & 16.42 \\
\hline 13 HARVARD & $2,114,111$ & $10,204,304$ & 20.72 & 44 & BOSTON & 970,155 & $4,958,895$ & 19.56 \\
\hline 14 PENNSYLVANIA & $2,103,118$ & $7,498,556$ & 28.05 & 45 & VIRGINIA & 855,024 & $5,350,099$ & 15.98 \\
\hline 15 WASHINGTON U.-ST. LOUIS & $2,043,251$ & $7,956,611$ & 25.68 & 46 & FLORIDA STATE & 854,074 & $5,808,581$ & 14.70 \\
\hline 16 COLUMBia & $1,878,538$ & $11,926,816$ & 15.75 & 47 & IOWA & 851,428 & $7,665,973$ & 11.11 \\
\hline 17 MONTREAL & $1,732,742$ & $7,232,096$ & 23.96 & 48 & UTAH & 832,417 & $4,500,465$ & 18.50 \\
\hline 18 ROCHESTER & $1,715,407$ & $3,741,503$ & 45.85 & 49 & MICHIGAN & 814,318 & $10,437,821$ & 7.80 \\
\hline 19 PENNSYLVANIA STATE & $1,675,811$ & $11,159,991$ & 15.02 & 50 & CALIFORNIA, IRVINE & 646,791 & $5,389,920$ & 12.00 \\
\hline 20 MIAMI & $1,669,551$ & $9,391,680$ & 17.78 & 51 & SOUTH CAROLINA & 588,072 & $4,862,112$ & 12.09 \\
\hline 21 CONNECTICUT & $1,639,340$ & $7,174,384$ & 22.85 & 52 & TULANE & 586,319 & $5,648,319$ & 10.38 \\
\hline 22 GEORGETOWN & $1,612,000$ & $5,003,140$ & 32.22 & 53 & LOUISIANA STATE & 406,400 & $4,099,957$ & 9.91 \\
\hline 23 GEORGE WASHINGTON & $1,602,048$ & $5,216,058$ & 30.71 & 54 & HAWAII & 405,404 & $5,636,156$ & 7.19 \\
\hline 24 SUNY-STONY BROOK & $1,586,252$ & $4,475,822$ & 35.44 & 55 & OKLAHOMA STATE & 269,290 & & \\
\hline 25 ILLINOIS, CHICAGO & $1,530,825$ & $1,530,825$ & 100.00 & 56 & SOUTHERN ILLINOIS & 141,866 & $4,261,789$ & 3.33 \\
\hline 26 OKLAHOMA & $1,502,046$ & $5,066,452$ & 29.65 & 57 & ALABAMA & 76,439 & $5,735,897$ & 1.33 \\
\hline 27 CASE WESTERN RESERVE & $1,494,041$ & $3,818,943$ & 39.12 & 58 & OHIO STATE & 5,458 & $5,598,596$ & 0.10 \\
\hline 28 CALIFORNIA, SAN DIEGO & $1,466,190$ & $5,772,873$ & 25.40 & 59 & CALIFORNIA, LOS ANGELES & & $5,150,666$ & \\
\hline 29 TENNESSEE & $1,463,420$ & $7,676,038$ & 19.06 & 59 & FLORIDA & . & $4,498,063$ & \\
\hline 30 WISCONSIN & $1,448,502$ & $6,513,942$ & 22.24 & 59 & MCMASTER & & $4,102,720$ & \\
\hline 31 KENTUCKY & $1,433,980$ & $6,285,343$ & 22.81 & 59 & SASKATCHEWAN & . & $4,321,026$ & \\
\hline
\end{tabular}





\section{ARL Statistics Questionnaire 2008-2009 \\ InSTRUCTIONS FOR COMPLETING THE QUeSTIONNAIRE}

\section{$\underline{\text { General Instructions }}$}

Definitions of statistical categories can be found in NISO Z39.7-2004, Information Services and Use: Metrics \& statistics for libraries and information providers--Data Dictionary (http://www.niso.org/). ARL has been modifying the interpretation of the standard definitions to address questions posed by library staff at various member institutions that complete the survey and with feedback from the ARL Statistics and Assessment Committee (http://www.arl.org/stats/aboutstats/index.shtml).

Please do not use decimals. All figures should be rounded to the nearest whole number.

Please respond to every question. If an exact figure cannot be provided, use NA/UA to indicate that the figure is either unavailable or not applicable. If the appropriate answer is zero or none, use $\mathbf{0 .}$

Although the form allows for data to be entered from both main and branch campuses, an effort should be made to report figures for the main campus only. (The U.S. National Center for Education Statistics, Integrated Postsecondary Education Data System (IPEDS) defines a branch institution as "a campus or site of an educational institution that is not temporary, is located in a community beyond a reasonable commuting distance from its parent institution, and offers organized programs of study, not just courses"). If figures for libraries located at branch campuses are reported, please specify which branch libraries are included and which ones are excluded in the notes below.

A branch library is defined as an auxiliary library service outlet with quarters separate from the central library of an institution, which has a basic collection of books and other materials, a regular staffing level, and an established schedule. A branch library is administered either by the central library or (as in the case of some law and medical libraries) through the administrative structure of other units within the university. Departmental study/reading rooms are not included.

The questionnaire assumes a fiscal year ending June 30, 2009. If your fiscal year is different, please indicate this in the notes below by adjusting the reporting period.

Footnotes. Explanatory footnotes will be included with the published statistics. Provide any notes you may have in the footnotes area at the end of the survey. Reporting libraries are urged to record there any information that would clarify the figures submitted in that line, e.g., the inclusion and exclusion of branch campus libraries. Please make an effort to word your footnotes in a manner consistent with notes appearing in the published report, so that the ARL Office can interpret your footnotes correctly. Please use a concise sentence/paragraph format when writing footnotes - do not use "bullets" or make a "bullet list."

\section{$\underline{\text { Specific Instructions }}$}

\section{Questions 1-1b. Volumes:}

Question 1. Volumes in Library. Use the ANSI/NISO Z39.7-2004 definition for volume as follows:

a single physical unit of any printed, typewritten, handwritten, mimeographed, or processed work, distinguished from other units by a separate binding, encasement, portfolio, or other clear distinction, which has been cataloged, classified, and made ready for use, and which is typically the unit used to charge circulation transactions. Either a serial volume is bound, or it comprises the serial issues that would be bound together if the library bound all serials. 
Include duplicates and bound volumes of periodicals. For purposes of this questionnaire, unclassified bound serials arranged in alphabetical order are considered classified. Exclude microforms, maps, nonprint materials, and uncataloged items. If any of these items cannot be excluded, please provide an explanatory footnote

Include government document volumes that are accessible through the library's catalogs regardless of whether they are separately shelved. "Classified" includes documents arranged by Superintendent of Documents, CODOC, or similar numbers. "Cataloged" includes documents for which records are provided by the library or downloaded from other sources into the library's card or online catalogs. Documents should, to the extent possible, be counted as they would if they were in bound volumes (e.g., 12 issues of an annual serial would be one or two volumes). Title and piece counts should not be considered the same as volume counts. If a volume count has not been kept, it may be estimated through sampling a representative group of title records and determining the corresponding number of volumes, then extrapolating to the rest of the collection. As an alternative, an estimate may be made using the following formulae:

\section{2 documents pieces per foot \\ 10 "traditional" volumes per foot \\ 5.2 documents pieces per volume}

Include e-book units, as long as these e-books are owned or leased and have been cataloged by your library. Include electronic books purchased through vendors such as NetLibrary ${ }^{\circledR}$ or Books $24 x 7$, and e-books that come as part of aggregate services. Include individual titles of e-book sets that are treated as individual reference sources. Include locally digitized electronic books and electronic theses and dissertations. Provide a footnote explaining how many e-books you are reporting, preferably by specifying the products and the number of titles in a note.

Include volumes purchased collectively where the cost is shared at the time of purchase.

If either formulas or sampling are used for deriving your count, please indicate in a footnote.

Question 1b. Volumes Added. Include only volumes cataloged, classified, and made ready for use. Include government documents if they have been included in the count of volumes on line 1a. Do not include as part of Volumes Added Gross any government documents or other collections (such as large gift collections or e-book packages, EBBO, etc.) that were added to the collection as the result of a one time download or addition to the OPAC. Include these items in Volumes Held of the previous year (Line 1a) and provide a footnote explaining the revision of Line 1a.

Question 2. Titles Held. Use the ANSI/NISO Z39.7-2004 definition for title as follows:

The designation of a separate bibliographic whole, whether issued in one or several volumes .... Titles are defined according to the Anglo-American Cataloging Rules. A book or serial title may be distinguished from other such titles by its unique International Standard Book Number (ISBN) or International Standard Serial Number (ISSN). This definition applies equally to print, audiovisual, and other library materials. For unpublished works, the term is used to designate a manuscript collection or an archival record series. Two subscriptions to Science magazine, for example, are counted as one title. When vertical file materials are counted, a file folder is considered a title.

Report the total number of unique titles cataloged, classified and made ready for use. The number of titles reported here is for the number of volumes reported under line (1). Include e-books as specified above in question (1). For those reporting a bibliographic volume under line (1), their title count may be exactly the same as their volume count. 
Question 3. Monographic Volumes Purchased. Report number of volumes purchased; do not include volumes received or cataloged. Include all volumes for which an expenditure was made during 2008-09, including volumes paid for in advance but not received during the fiscal year. Include monographs in series and continuations. Include e-books that fit the NetLibrary ${ }^{\circledR}$ model, i.e., electronic manifestations of physical entities and/or units; provide a footnote explaining how many e-books you are reporting, preferably by specifying the products and the number of titles. If only number of titles purchased can be reported, please report the data and provide an explanatory footnote.

Question 4: Basis of Volume Count. A physical count is a piece count; a bibliographic count is a catalog record count.

Questions 5. Serials. Use the following definition adapted from AACR2 for a serial:

A bibliographic resource issued in a succession of discrete parts, usually bearing numbering, that has no predetermined conclusion. Examples of serials include journals, magazines, electronic journals, continuing directories, annual reports, newspapers, and monographic series.

Report the total number of unique serial titles, NOT SUBSCRIPTIONS, that you currently acquire and to which you provide access. Do not include duplicate counts of serial titles. Report each title once, regardless of how many subscriptions or means of access you provide for that title. Exclude unnumbered monographic and publishers' series. Electronic serials acquired as part of a bundle or an aggregated package should be counted at the title level, even if they are not cataloged, as long as the title is made accessible directly by the library (e.g., through a finding aid). If access is provided only through the overall platform or aggregator, do not report the individual titles but count the package as a single title.

Question 5a. Serial titles currently purchased. In the case of consortial agreements, count under 'serial titles currently purchased' those titles for which the library pays any amount from its budgeted expenditures. Include all titles that are part of bundles or aggregated packages, even if your library makes a partial payment for access to those titles. If a purchased title includes electronic access to the title, count that title ONLY ONCE (DEDUPED) as electronic only. If a database includes full-text and abstracted titles, the number of full-text titles can be counted.

Question 5b. Serial titles: Not Purchased. Report other titles that your library receives and does not pay for directly under 'serial titles received but not purchased.' These titles may include exchanges, gifts, etc.

If serial titles have been purchased through a consortium whose budget is centrally funded and independent from the library's budget, these serials should be reported under 'serial titles currently received but not purchased.' If within a purchased or aggregated package it cannot be determined that some titles are not purchased, report all titles as purchased.

Freely accessible titles are those your library provides direct access to via cataloging records or through online serial lists of other finding aids.

To the extent possible, report all government document serials separately in (5b.iv).

If separate counts of non-purchased and purchased serial titles are not available, report only the total number of serial titles currently purchased and received on line (5), and report NA/UA for lines (5a) and (5b).

Question 7. Microforms. Report the total number of physical units: reels of microfilm, microcards, and microprint and microfiche sheets. Include all government documents in microform; provide a footnote if documents are excluded. 
Question 8. Government documents. Report the total number of physical units (pieces) of government documents in paper format that have not been counted elsewhere. Include local, state, national, and international documents; include documents purchased from a commercial source if shelved with separate documents collections and not counted above. Include serials and monographs. To estimate pieces from a measurement of linear feet, use the formula 1 foot $=52$ pieces and indicate in a footnote that the count is based on this estimate. Exclude microforms and non-print formats such as maps or CD-ROMs. Adjust line (1a), i.e., last year's Volumes Held, and provide a footnote if you are adding records to the OPAC for government documents previously held but not counted as part of Volumes Held line (1a).

Question 9. Computer files. Include the number of pieces of computer-readable disks, tapes, CD-ROMs, and similar machinereadable files comprising data or programs that are locally held as part of the library's collections available to library clients. Examples are U.S. Census data tapes, sample research software, locally-mounted databases, and reference tools on CD-ROM, tape or disk. Exclude bibliographic records used to manage the collection (i.e., the library's own catalog in machine-readable form), library system software, and microcomputer software used only by the library staff.

Question 10. Manuscripts and archives. Include both manuscripts and archives measured in linear feet.

Question 11. Cartographic materials. Include the numbers of pieces of two- and three-dimensional maps and globes. Include satellite and aerial photographs and images.

Question 12. Graphic materials. Include the number of pieces of prints, pictures, photographs, postcards, slides, transparencies, film strips, and the like.

Question 13. Audio materials. Include the number of pieces of audiocassettes, phonographic discs, audio compact discs, reel-toreel tapes, and other sound recordings.

Question 14. Film and video materials. Include the number of pieces of motion pictures, videocassettes, video laser discs, and similar visual materials.

Questions 15-21. Expenditures. Report all expenditures of funds that come to the library from the regular institutional budget, and from sources such as research grants, special projects, gifts and endowments, and fees for service. (For question (18), include nonlibrary funds; see instruction for question (18). Do not report encumbrances of funds that have not yet been expended. Canadian libraries should report expenditures in Canadian dollars. (For your information, if interested in determining figures in U.S. dollars, divide Canadian dollar amounts by 1.1667, the average monthly noon exchange rate published in the Bank of Canada Review for the period July 2008-June 2009). Please round figures to the nearest dollar.

Question 16a. Monographs. Report expenditures for volumes purchased counted on line (3).

Question 16b. Serial titles. Report expenditures for serial titles counted on line (5a). Exclude unnumbered monographic and publishers' series, and encumbrances.

Question 16c. Other library materials. Include expenditures for all materials not reported in Questions (16a) and (16b), e.g., backfiles of serials, charts and maps, audiovisual materials, manuscripts, etc. If expenditures for these materials are included in lines (16a) and/or (16b) and cannot be disaggregated, please report U/A and provide a footnote. Do not include encumbrances.

Question 16d. Miscellaneous expenditures. Include any other materials funds expenditures not included in questions (16a)-(16c), e.g., expenditures for bibliographic utilities, literature searching, security devices, memberships for the purposes of publications, etc. Please list categories, with amounts, in a footnote. Note: If your library does not use materials funds for non- 
materials expenditures - i.e., if those expenditures are included in "Other Operating Expenditures" - report 0, not NA/UA, on line (16d).

Question 17. Contract Binding. Include only contract expenditures for binding done outside the library. If all binding is done inhouse, state this fact and give in-house expenditures in a footnote; do not include personnel expenditures. Note: this figure should also be reported in the 2008-09 ARL Preservation Survey, question (8b).

Questions 18. Salaries and wages. Exclude fringe benefits. If professional, support staff and student salaries cannot be separated, enter NA/UA, in lines (18a), (18b) and (18c) and enter total staff salaries in line (18).

Question 18c. Salaries and wages: Student Assistants. Report 100\% of student wages regardless of budgetary source of funds. Include federal and local funds for work study students.

Question 20. Other operating expenditures. Exclude expenditures for buildings, maintenance, and fringe benefits.

Questions 22-26. Electronic expenditures. These items are intended to indicate what portion of your institution's total library expenditures are dedicated to electronic resources and services. Please use the Footnotes to indicate any electronic materials expenditures you believe not to be covered by these questions. Many expenditures recorded in these questions should have been included in question (21), total library expenditures.

Question 22. One-time electronic resource purchases. Report expenditures that are not current serials (i.e. are nonsubscription, one-time, or monographic in nature) for software and machine-readable materials considered part of the collections. Examples include periodical backfiles, literature collections, one-time costs for JSTOR membership, etc. Expenditures reported here may be derived from any of the following categories: Monographs (16a), Other Library Materials (16c), Miscellaneous (16d), or Other Operating Expenditures (20).

Question 23. Ongoing electronic resource purchases. Report subscription expenditures (or those which are expected to be ongoing commitments) for serial publications whose primary format is electronic and for online searches of remote databases such as OCLC FirstSearch, DIALOG, Lexis-Nexis, etc. Examples include paid subscriptions for electronic journals and indexes/abstracts available via the Internet, CD-ROM serials, and annual access fees for resources purchased on a "one-time" basis, such as literature collections, JSTOR membership, etc. Not all items whose expenditures are counted here will be included in Serial titles currently received question (5) or Serials Expenditures question (16b).

Question 24. Bibliographic Utilities, Networks, and Consortia. Because it is increasingly common for ARL Libraries to enter into consortial arrangements to purchase access to electronic resources, both "Library" and "External" expenditure blanks and instructions are provided. Please use afootnote to describe expenditures that you believe are not covered by the question, or situations that do not seem to fit the instructions.

Question 24a. From internal library sources. Report expenditures paid by the Library for services provided by national, regional, and local bibliographic utilities, networks, and consortia, such as OCLC and RLG, unless for user database access and subscriptions, which should be reported in questions (22) or (23). Include only expenditures that are part of Other Operating Expenditures (Q20).

Question 24b. From external sources. If your library receives access to computer files, electronic serials or search services through one or more centrally-funded system or consortial arrangements for which it does not pay fully and/or directly (for example, funding is provided by the state on behalf of all members), enter the amount paid by external bodies on its behalf. If the specific dollar amount is not known, but the total student FTE for the consortium and amount spent for the academic members are known, divide the overall amount spent by your institution's share of the total student FTE. 
Question 25. Computer hardware and software. Report expenditures from the library budget for computer hardware and software used to support library operations, whether purchased or leased, mainframe or microcomputer, and whether for staff or public use. Include expenditures for: maintenance; equipment used to run information service products when those expenditures can be separated from the price of the product; telecommunications infrastructure costs, such as wiring, hubs, routers, etc. Include only expenditures that are part of Other Operating Expenditures (20).

Question 26. Document Delivery/Interlibrary Loan. Report expenditures for document delivery and interlibrary loan services (both borrowing and lending). Include fees paid for photocopies, costs of telefacsimile transmission, royalties and access fees paid to provide document delivery or interlibrary loan. Include fees paid to bibliographic utilities if the portion paid for interlibrary loan can be separately counted. Include only expenditures that are part of Miscellaneous Materials Expenditures (16d) or Other Operating Expenditures (20), and only for those ILL/DD programs with data recorded in Questions (35)-(36).

Questions 27. Personnel. Report the number of FTE (full-time equivalent) staff in filled positions, or positions that are only temporarily vacant. ARL defines temporarily vacant positions as positions that were vacated during the fiscal year for which ARL data were submitted, for which there is a firm intent to refill, and for which there are expenditures for salaries reported on line (18).

Include cost recovery positions and staff hired for special projects and grants, but provide an explanatory footnote indicating the number of such staff. If such staff cannot be included, provide a footnote. To compute full-time equivalents of part-time employees and student assistants, take the total number of hours per week (or year) worked by part-time employees in each category and divide it by the number of hours considered by the reporting library to be a full-time work week (or year). Round figures to the nearest whole numbers.

Question 27a. Professional Staff. Since the criteria for determining professional status vary among libraries, there is no attempt to define the term "professional." Each library should report those staff members it considers professional, including, when appropriate, staff who are not librarians in the strict sense of the term, for example computer experts, systems analysts, or budget officers.

Question 27b. Support Staff. Report the total FTE (see instruction (27) of staff not included in (27a).

Question 27c. Student Assistants. Report the total FTE (see instruction Q27) of student assistants employed on an hourly basis whose wages are paid from funds under library control or from a budget other than the library's, including federal work-study programs. Exclude maintenance and custodial staff.

Question 28. Number of staffed library service points. Count the number of staffed public service points in the main library and in all branch libraries reported in this inventory, including reference desks, information desks, circulation, current periodicals, reserve rooms, reprographic services (if staffed as a public facility), etc. Report the number of designated locations, not the number of staff.

Question 29. Number of weekly public service hours. Report an unduplicated count of the total public service hours per typical full-service week (i.e., no holidays or other special accommodations) across both main library and branches using the following method (corresponds to IPEDS): If a library is open from 9:00 a.m. to 5:00 p.m. Monday through Friday, it should report 40 hours per week. If several of its branches are also open during these hours, the figure remains 40 hours per week. Should Branch A also be open one evening from 7:00 p.m. to 9:00 p.m., the total hours during which users can find service somewhere within the system becomes 42 hours per week. If Branch B is open the same hours on the same evening, the count is still 42, but if Branch B is open two hours on another evening, or remains open two hours later, the total is then 44 hours per week. Exclude 24-hour unstaffed reserve or similar reading rooms. The maximum total is 168 (i.e., a staffed reading room open 7 days per week, 24 hours per day).

Questions 30-31. Instruction. Sampling based on a typical week may be used to extrapolate TO A FULL YEAR for Questions (30) and (31). Please indicate if responses are based on sampling. 
Question 30. Presentations to Groups. Report the total number of sessions during the year of presentations made as part of formal bibliographic instruction programs and through other planned class presentations, orientation sessions, and tours. If the library sponsors multi-session or credit courses that meet several times over the course of a semester, each session should be counted. Presentations to groups may be for either bibliographic instruction, cultural, recreational, or educational purposes. Presentations both on and off the premises should be included as long as they are sponsored by the library. Do not include meetings sponsored by other groups using library meeting rooms. Do not include training for library staff; the purpose of this question is to capture information about the services the library provides for its clientele. Please indicate if the figure is based on sampling.

Question 31. Participants in Group Presentations. Report the total number of participants in the presentations reported on line (30). For multi-session classes with a constant enrollment, count each person only once. Personal, one-to-one instruction in the use of sources should be counted as reference transactions on line (32). Please indicate if the figure is based on sampling. Use a footnote to describe any special situations.

Question 32. Reference Transactions. Report the total number of reference transactions. A reference transaction is

an information contact that involves the knowledge, use, recommendations, interpretation, or instruction in the use of one or more information sources by a member of the library staff. The term includes information and referral service. Information sources include (a) printed and nonprinted material; (b) machine-readable databases (including computer-assisted instruction); (c) the library's own catalogs and other holdings records; (d) other libraries and institutions through communication or referral; and (e) persons both inside and outside the library. When a staff member uses information gained from previous use of information sources to answer a question, the transaction is reported as a reference transaction even if the source is not consulted again.

If a contact includes both reference and directional services, it should be reported as one reference transaction. Include virtual reference transactions (e.g., e-mail, WWW form, chat). Duration should not be an element in determining whether a transaction is a reference transaction. Sampling based on a typical week may be used to extrapolate TO A FULL YEAR for Question 32 . Please indicate if the figure is based on sampling.

EXCLUDE SIMPLE DIRECTIONAL QUESTIONS. A directional transaction is an information contact that facilitates the logistical use of the library and that does not involve the knowledge, use, recommendations, interpretation, or instruction in the use of any information sources other than those that describe the library, such as schedules, floor plans, and handbooks.

Questions 33-34. Circulation. For Question (33), count the number of initial circulations during the fiscal year from the general collection for use usually (although not always) outside the library. Do not count renewals. Include circulations to and from remote storage facilities for library users (i.e., do not include transactions reflecting transfers or stages of technical processing). Count the total number of items lent, not the number of borrowers.

For Question (34), report total circulation for the fiscal year including initial transactions reported on line (33) and renewal transactions. Exclude reserve circulations; these are no longer reported.

Questions 35-36. Interlibrary Loans. Report the number of requests for material (both returnables and non-returnables) provided to other libraries on line (35) and the number of filled requests received from other libraries or providers on line (36). On both lines, include originals, photocopies, and materials sent by telefacsimile or other forms of electronic transmission. Include patron-initiated transactions. Exclude requests for materials locally owned and available on the shelves or electronically. Do not include transactions between libraries covered by this questionnaire. 
Question 37. PhD Degrees. Report the number awarded during the 2007-08 fiscal year. Please note that only the number of Ph.D. degrees are to be counted. Statistics on all other advanced degrees (e.g., D.Ed., D.P.A., M.D., J.D.) should not be reported in this survey. If you are unable to provide a figure for Ph.D.s only, please add a footnote.

Question 38. PhD Fields. For the purposes of this report, Ph.D. fields are defined as the specific discipline specialties enumerated in the U.S. Department of Education's Integrated Postsecondary Education Data System (IPEDS) “Completions" Survey. Although the IPEDS form requests figures for all doctoral degrees, only fields in which PhDs are awarded should be reported on the ARL questionnaire. Any exceptions should be footnoted.

Question 39. Instructional Faculty. Instructional faculty are defined by the U.S. Dept. of Education as:

members of the instruction/research staff who are employed full-time as defined by the institution, including faculty with released time for research and faculty on sabbatical leave.

Full-time counts generally exclude faculty who are employed to teach fewer than two semesters, three quarters, two trimesters, or two four-month sessions; replacements for faculty on sabbatical leave or leave without pay; faculty for preclinical and clinical medicine; faculty who are donating their services; faculty who are members of military organizations and paid on a different pay scale from civilian employees; academic officers, whose primary duties are administrative; and graduate students who assist in the instruction of courses. Please be sure the number reported, and the basis for counting, are consistent with those for 2006-07 (unless in previous years faculty were counted who should have been excluded according to the above definition). Please footnote any discrepancies.

Questions 40-43. Enrollment. U.S. libraries should use the Fall 2008 enrollment figures reported to the Department of Education on the Integrated Postsecondary Education Data System survey. Please check these figures against the enrollment figures reported to ARL last year to ensure consistency and accuracy. Note: In the past, the number of part-time students reported was FTE; the number now reported to IPEDS is a head count of part-time students. Canadian libraries should note that the category "graduate students" as reported here includes all post-baccalaureate students.

\section{FOOTNOTES}

Please consult the data entry Web interface (www.arlstatistics.org) for a copy of last year's footnotes. These can be found under "Data Repository" after you login into www.arlstatistics.org. Explanatory footnotes will be included with the published statistics. Reporting libraries are urged to record in the footnote section any information that would clarify the figures submitted, e.g., the inclusion and exclusion of branch campus libraries (see the "General Instructions" for definition of branch campus libraries). Please make an effort to word your footnotes in a manner consistent with notes appearing in the published report, so that the ARL Office can interpret your footnotes correctly.

NOTE: Any change over 10\% in any answer to any of the survey's questions over the preceding year's response (2007-08) should be addressed with a footnote.

Submit the completed questionnaire by

October 15, 2009.

For assistance, please e-mail Martha Kyrillidou (martha@arl.org) or Les Bland (les@arl.org) Tel. (202) 296-2296. 


\section{ARL STATISTICS WORKSHEET 2008-2009}

This worksheet is designed to help you plan your submission for the 2008-2009 ARL Statistics. The figures on this worksheet should be similar to those in the "Summary" page of your web form, except in cases where data are unavailable. If an exact figure is unavailable, use "NA/UA". If the appropriate answer is zero or none, use " 0. "

Reporting Institution Date Returned to ARL

Report Prepared by (name)

Title

Email address Phone number

Contact person (if different)

Title

Email address Phone number

PAGE ONE - VOLUMES AND TITLES:

1. Volumes held June 30, 2009 (1.a $+1 . b)$

(1)

1a. Volumes held June 30, 2008

(1.a)

1b. Volumes added during the year (1.b.i - 1.b.ii)

(i) Volumes added - Gross

(ii) Volumes withdrawn during year

(1.b.ii)

2. Titles held June 30, 2009

(2)

3. Number of monographic volumes purchased

(3)

4. Basis of volume count is:

(4)

Physical

Bibliographic 
PAGE TWO - OTHER COLLECTIONS

SERIALS

5. Total number of serial titles currently received, including periodicals $(5 . a+5 . b)$

(5)

5a. Number of serial titles currently purchased $(5 a . i+5 a . i i)(5 a)$

5a.i Electronic

5a.ii Print (and other format) serials purchased

(5a.ii)

$5 b$. Number of serial titles currently received but not purchased

$$
(5 b . i+5 b . i i+5 b . i i i+5 b . i v)
$$

5b.i Consortial

5b.ii Freely accessible

5b.iii Print (and other format) - Exchanges, gifts, etc.

(5b.iii)

5b.iv Government documents

(5b.iv)

6. Government documents are included in count of Current Serials?

(6) Yes No

\section{OTHER LIBRARY MATERIALS}

7. Microform units

(7)

8. Government documents not counted elsewhere

(8)

9. Computer files

(9)

10. Manuscripts and archives (linear ft.)

(10)

\section{AUDIOVISUAL MATERIALS}

11. Cartographic

12. Graphic

13. Audio

14. Film and Video 
PAGE THREE - EXPENDITURES

15. Are the below figures reported in Canadian dollars?

(15) Yes

No

16. Total Library Materials Expenditures $(16 \cdot a+16 . b+16 . c+16 . d)$

(16)
16a. Monographs
(16a)
16b. Serial titles, including periodicals
(16b)
16c. Other Library Materials
(16c)
16d. Miscellaneous
(16d)

17. Contract binding

(17)

18. Total Salaries and Wages $(18 . a+18 . b+18 . c)$

(18)

18a. Professional staff

(18a)

18b. Support staff

18c. Student assistants

(18c)

19. Fringe benefits are included in expenditures for salaries and wages? (19) Yes No

20. Other operating expenditures

(20)

21. Total library expenditures $\quad(16+17+18+20)$

(21)

\section{ELECTRONIC MATERIALS EXPENDITURES}

22. One-time electronic resource purchases

23. Ongoing electronic resource purchases (e.g., subscriptions, annual license fees)

(23)

24. Bibliographic Utilities, Networks, and Consortia

24a. From internal library sources

(24a)

24b. From external sources

25. Computer hardware and software

(25)

26. Document Delivery/Interlibrary Loan

(26) 
PAGE FOUR - PERSONNEL AND PUBLIC SERVICES

PERSONNEL (Round figures to nearest whole number.)

27. Total Staff FTE $(27 . a+27 . b+27 . c)$

27a. Professional staff, FTE

(27a)

27b. Support staff, FTE

(27b)

27c. Student assistants, FTE

$(27 \mathrm{c})$

\section{STAFFED SERVICE POINTS AND HOURS}

28. Number of staffed library service points

(28)

29. Number of weekly public service hours

(29)

\section{INSTRUCTION}

30. Number of library presentations to groups

(30)

30a. Is the library presentations figure based on sampling?

(30a) Yes No

31. Number of total participants in group presentations reported in line 30

31a. Is the total participants in group presentations figure based on sampling?

(31a) Yes No

\section{REFERENCE}

32. Number of reference transactions

(32)

32a. Is the reference transactions figure based on sampling?

(32a) Yes No 


\section{CIRCULATION}

33. Number of initial circulations (excluding reserves)

34. Total circulations (initial and renewals, excluding reserves)

\section{INTERLIBRARYLOANS}

\section{Total number of filled requests provided to other libraries}

36. Total number of filled requests received from other libraries or providers

\section{PhD DEGREES AND FACULTY}

37. Number of PhDs awarded in FY2008-2009

38. Number of fields in which PhDs can be awarded

39. Number of full-time instructional faculty in FY2008-2009

ENROLLMENT - FALL 2008

(Line numbers refer to IPEDS survey form.)

40. Full-time students, undergraduate and graduate

(Add line 8, columns $15 \mathcal{E} 16$, and line 14, columns $15 \mathcal{E} 16$. .)

41. Part-time students, undergraduate and graduate

(Add line 22, columns $15 \& 16$, and line 28, columns $15 \& 16$.)

43. Part-time graduate students (Line 28, columns $15 \mathcal{E} 16$.

\section{FOOTNOTES}

On the web form, you will be able to add footnotes to individual questions, as well as footnotes that apply to your entire institution. Please provide any information which would clarify the figures submitted, e.g., the inclusion of branch campus libraries or any special projects which might cause radical increases or decreases. Please use the footnotes in the ARL Statistics 2007-2008 for comparison if necessary. Please consult the Data Repository under www.arlstatistics.org for a copy of last year's footnotes. These can be found under "Data Repository" after you login to www.arlstatistics.org. Please make an effort to word your footnotes in a manner consistent with notes appearing in the published report, so that the ARL Office can interpret your footnotes correctly. Please use a concise sentence/paragraph format when writing footnotes-do not use bullets or make a bullet list.

NOTE: Any change over 10\% in any answer to any of the survey's questions over the preceding year's response (2007-08) should be addressed with a footnote.

\section{Submit the completed questionnaire by}

\section{October 15, 2009.}

For assistance, please e-mail Martha Kyrillidou (martha@arl.org) or Les Bland (les@arl.org) Tel. (202) 296-2296. 



\section{FOOTNOTES}

Footnotes may also include errata and corrections to data not previously reported from prior years. Numbers refer to columns in Library Data Tables and to Questionnaire numbers. Unless otherwise noted, all figures are as of 06/30/2009.

QuESTION FOOTNOTE

NUMBER

ALABAMA

All figures are as of 09/30/2009.

\section{ARIZONA}

Library branches included: Arizona Health Sciences Library-Phoenix.

24.a AMIGOS/OCLC charges were $\$ 8,410$ : membership $\$ 350$, user fee $\$ 1,100$, annual usage $\$ 6,960$. However, the amount shown was covered by cataloging and ILL credits held at AMIGOS.

30-31 Includes education and outreach group teaching.

\section{CALIFORNIA, DAVIS}

Library branches included: Blaisdell and Carlson Health Sciences Libraries.

Library branches NOT included: Shields Library and the Physical Science and Engineering Library

1.a Volumes held June 30, 2008 revised to 370,836.

\section{CALIFORNIA, LOS ANGELES}

5.a Serial count is an estimate based on a manual count, a total of 3,195 titles. The Serial count as reported in General Library is for all units and includes Health Sciences.

\section{CALIFORNIA, SAN DIEGO}

Library branches included: Biomedical Library and Medical Center Library.

Data for questions 28-43 are included in the Main Library reporting.

1.a Volumes held June 30, 2008 revised to 240,708.

28-31 Counted in the Main Library statistics.

\section{CASE WESTERN RESERVE}

1.b.i The Health Sciences Library has access to 13,091 e-books that are reported in the main library's statistics.

5.a.i The Health Sciences Library has access to 59,448 e-journals that are reported in the main library's statistics.

\section{CHICAGO}

John Crerar Library is a central science library with a biomedical collection for health sciences. Many numbers are percentages as a result. Data for the ARL survey are matched as closely as possible to data compiled and reported for the Association of Academic Health Sciences Libraries' (AAHSL) Annual Statistics of Medical School Libraries in the United States and Canada.

5-5.a.ii Figure reflects new, improved method for more accurate estimate of proportion of total number serial titles that are biomedical titles.

16.a-16.b While there are fluctuations within the categories, overall spending was up only $9.92 \%$. Serials print cancellations and added electronic purchases may account for some of the individual variation.

18-18.c Includes fringe benefits.

22 One-time purchases may vary significantly from year to year depending on availability of discretionary funds for biomedical uses.

23 Figure reflects cost increases for existing resources and the addition of new subscription products.

27 Net reduction in staffing due to budget reductions.

27.a New librarian position added.

27.b Reduction in support staffing due to budget reductions. 


\section{CHICAGO, cont}

30-31 New librarian position added with emphasis in instruction and outreach.

\section{CINCINNATI}

1.b Decrease reflects the weeding of the Nursing Library collection plus weeding of some bound journals in the Health Sciences Library.

1.b.ii As in 1.b, this large number of withdrawals reflects the weeding of the Nursing Library collection plus weeding of some bound journals in the Health Sciences Library.

5 Serials not reported separately from total university count.

17 Decrease reflects fewer print journals purchased and more careful attention to not binding when archival rights are held to an electronic journal.

18, 25, 27 Decrease reflects the completion of the reorganization that separated what was formerly a combined HSL and IT organization. The HSL is now integrated into the central library system administratively and operationally as it originally was until 1972.

37 PhD degrees, faculty, and enrollment are not reported separately from total university counts.

\section{COLUMBIA}

$5 \quad$ Health Sciences serials data included in Butler Library statistics.

12 Wilcox Collection

26 Document Delivery / Interlibrary Loan figures included in Butler Library statistics.

\section{CONNECTICUT}

1.b.i Includes 1,301 e-books.

\section{CORNELL}

1.a Volumes held June 30, 2008 revised to 200,301. This year's increase reflects additional available volumes, and the fact that the 2007-2008 e-book count was significantly underreported. The e-book count may include a small amount of duplication between e-book packages yet to be determined.

1.b.i Includes some manually cataloged e-books.

2 The count may include a small amount of duplication between e-book packages yet to be determined.

5 Estimate only. $2.5 \%$ of the titles in this count were estimated to be print. 1,430 of the e-titles were estimated to be noncurrent. Some deduping done through estimates. Decrease in print title count due to cancellations to favor electronic format over print. Increase in e-title count reflects additional available titles (including the selective addition of records for open access titles), and a more precise counting methodology.

7 Starting with 2008-2009, counts for microforms will no longer be kept up to date; reporting will be at the 2007-2008 level.

12 Starting with 2008-2009, graphics counts will no longer be kept up to date; reporting will be at the 2007-2008 level. The change reflects rounding only.

14 Starting with 2008-2009, motion picture counts will no longer be kept up to date; reporting will be at the 2007-2008 level. Video and DVD counts are now collected online vs. manually.

16.d Figure reflects shipping costs only.

$30 \quad$ Includes some instruction sessions for individuals.

32 Extrapolated from 12 random sampling weeks. In large part, increase reflects a greater effort to record all transactions.

33 In large part, the decrease reflects a counting error made in 2007-2008.

37 Includes 13 double major MDs and PhDs. The total for PhDs only is 58.

\section{DARTMOUTH}

Library branches included: Dana Biomedical Library and Matthews-Fuller Health Sciences Library.

1.b.i- Withdrawals part of ongoing deaccessioning project.

1.b.ii

5, 16.a Figure indicates budget constraints.

5.b.iii Reduced number of gifts and exchanges received and/or kept. 
DARTMOUTH, cont.

\section{EMORY}

Figure indicates move to electronic only.

Figure indicates deaccessioning project.

Figure reflects reduction in binding program.

Increase in Other Operating Expenditures is due to a significant increase in endowment distribution (50\%) in 2008-2009 and increased reserve spending during the year.

Figure indicates move to electronic only.

Figure reflects a low point in replacement cycle.

Figure reflects inaccuracies in 2007-2008 reporting.

Figure reflects staff reorganization.

Figure reflects increased emphasis in this area.

Figure correlates to expansion in electronic holdings.

All figures are as of 08/31/2009.

Library branches included: Grady Branch Library and Hospital Branch Library.

Library branches NOT included: Midtown Branch Library.

5.b Figure not reported in 2007-2008.

16.c Figure reflects the purchase of a backfile.

16.d There was a cost increase for one product. We also added a new product.

20 A portion of "Other operating expenditures" goes as an expense to carryforward account.

FLORIDA

16.a Most of this money was spent as an end of year deposit account. This means that the titles purchased will be counted in the 2009-2010 fiscal year.

\section{FLORIDA STATE}

Library branches NOT included: Main Library (Strozier), Dirac Science Library, College of Engineering Library, Allen Music Library, College of Law Research Center, Goldstein College of Information Library, Ringling Museum of Art Library, Panama City, Panama Library, and Panama City, Florida Library.

1.b.i Increase due to addition of a 3,532 volumes of bound serials previously owned but not counted.

16.b Represents a payment by the College of Medicine to contribute to an invoice for access for biomedical and medical journals subscribed to by University Libraries.

$30 \quad$ 2007-2008 presentation figure included only formal faculty development classes. 2008-2009 includes formal faculty development classes as well as library tours and orientations.

31 The number of participants for the 2007-2008 year included only formal faculty development classes. 2008-2009 number of participants includes formal faculty development classes as well as library tours and orientations.

Since the professional librarian staff was down by two for the period from July 2008 to June 2009, this figure is based on an average of totals from the previous two years. As six staff members provide reference services on a regular basis, this average was then multiplied by $4 / 6$ to estimate the number of reference transactions.

\section{GEORGE WASHINGTON}

1 Total physical volumes significantly reduced due to change in space availability requiring large reduction in physical volumes held; content change was minimal due to electronic availability.

1.a Volumes held June 30, 2008 revised to 130,499.

1.b The number withdrawn exceeded the number added resulting in a negative percent changed.

1.b.ii Large number of physical volumes withdrawn due to space availability change. Overall content loss was minimal.

5.a A different calculation method showed possible previous error. 


\section{GEORGE WASHINGTON, cont.}

13 Audio Digest audio tape collection weeded 2008-2009.

16.a Software was included in this figure in 2007-2008.

16.b Entire budget figure used for calculation this year.

16.c Backfiles expenditures are reported in Q22.

17 Binding was significantly reduced due to a loss of space.

18.c A large-scale project required the significant additional use of student assistants.

20 Fewer computer/networking purchases due to replacement cycle.

21 One of components significantly reduced resulting in overall sum change.

22 Backfiles were purchased this year.

26 ILL invoices paid were not included in the 2007-2008 figure in error. Also, costs of borrowing increased.

27.a The actual figure is not a full integer but is 13.5 .

27.b The correct figure is 16.5, but the system will not accept a decimal.

30 Course librarians routinely present in was reduced in number of sessions by $25 \%$.

32 Recording lapses due to staff turnover.

35 Decrease due to removal of many bound journals from collection.

36 Increase due to internal removal of bound journals and decreased accessibility of remaining journal titles during remodeling project.

\section{GEORGETOWN}

1.b Decrease is the result of far fewer volumes purchased plus Spring 2009 weeding project.

1.b.ii Spring 2009 weeding project undertaken.

5.b.iii We are unable to break down the 1,008 serials reported here. The figure was placed here so that the operating system used to produce this report would correctly aggregate these numbers into our main 2008-2009 ARL Statistics.

22 No e-resource backfile runs purchased in 2008-2009.

HAWAII

1.a The number of e-books being reported is 5,683 .

26 This service is provided by the University of Hawaii at Manoa Library.

HOWARD

1.a Volumes held June 30, 2008 revised to 134,008.

\section{ILLINOIS, CHICAGO}

Library branches included: Library of the Health Sciences - Chicago, Peoria, Rockford, and Urbana campuses.

Library branches NOT included: Richard J. Daley Library, Science Library

1.a Volumes held June 30, 2008 revised to 741,475.

$40 \quad$ All student figures included in Daley statistics.

INDIANA

1.a Volumes held June 30, 2008 revised to 287,737 which includes the addition of a Springer e-book collection consisting of 13,000 volumes.

3 Includes 13,058 e-books.

29 Actually, 91.5 hours per week.

IOWA

1.a Volumes held June 30, 2008 revised to 350,558. 
KANSAS

1.b Decrease in volumes added due to systematic journal weeding and withdrawals.

5.b.ii Weeded our list of freely available titles to those with significant holdings and relevance, which decreased the number of freely accessible materials.

16.b Reallocated money from monographs to serials.

18.b We were able to add staffing, primarily through grant funding.

21 Increase in 2008-2009 over 2007-2008 figure primarily represents an infusion of grant funding.

\section{KENTUCKY}

3 Includes 169 e-books.

$5 \quad$ We are unable to breakout a separate electronic serial titles total for the health sciences. The Medical Center Library's electronic serial titles, both purchased and not purchased, are included in the total for the Main Library. The total reported here represents health sciences print serial titles only, both purchased and not purchased.

5.a Represents print serial titles only.

5.a.i The Medical Center Library's electronic serial titles (purchased) are included in the total for the Main Library. We are unable to breakout a separate total for the health sciences.

5.b The Medical Center Library's electronic serial titles (not purchased) are included in the total for the Main Library. We are unable to breakout a separate electronic serial titles total for the health sciences. The total reported here represents only the health sciences print serial titles (not purchased).

5.b.i The Medical Center Library's consortial serial titles (received but not purchased) are included in the total for the Main Library. We are unable to breakout a separate total for the health sciences.

5.b.ii The Medical Center Library's freely accessible serial titles (received but not purchased) are included in the total for the Main Library. We are unable to breakout a separate total for the health sciences.

\section{LOUISIANA STATE}

1.a

1.b.i-

1.b.ii, 3

5.a.i- $\quad$ Decrease due to new method of counting.

5.b.ii

5.b.iii

20

22,35

27

37

42

MCGILL

All figures are as of $05 / 31 / 2009$

1.a Volumes held revised to 495,757. This figure represents an adjusted total, which included the Olser, Biology, and Life Science libraries.

1.b.ii Increase due to the many volumes that were withdrawn as we now have online access.

3 Increase due to the purchased of a large set of e-books for Health Sciences -- Canadian Health Research Collection from Canadian Electronic Library.

5.a.i This is an estimate based on our SFX KB for titles we get from various vendors in the Health Sciences.

16-26 Expenditures as reported in Canadian dollars: (16a) \$549,171; (16b) \$1,396,112; (16c) \$9,085; (16d) \$0; (16) \$1,954,368; (17) \$0; (18a)\$721,470; (18b) \$461,384; (18c) \$32,014; (18) \$1,214,868; (20) \$94,900; (21) \$3,264,136; (22) \$72,662; (23) \$1,183,765; (24a) \$0; (24b) $\$ 0$; (25) \$0; (26) \$0.

The figure for the number of full-time instructional faculty was taken from the from the McGill factbook. 


\section{MCMASTER}

All figures are as of 04/30/2009.

5.a.i,

5.b.ii

37

$16-26$

MIAMI

9 Medical library computer files are reported in Q14.

14

18.a

27.a

27.b

$37-43$

\section{MICHIGAN}

1, 1.b-1.b.i

3

5.a

5.b

5.b.ii

5.b.iii

16

33

34

35-36

\section{MINNESOTA}

Library branches included: Veterinary Medical Library.

2

Breakdown not available.

3

previous years' reports.

5.a.i Unique title counts are reported at the institutional (University Libraries) level, not by branch.

12 Breakdown by type is not available.

24.a-24.b Included in Main Library expenditures.

37-43 Information for entire institution is reported with Main Library data. Breakdown not available.

\section{MONTREAL}

$16-26$

All figures are as of 05/31/2009.

Library branches NOT included: Bibliothèques UdeM, Droit (Law), École Polytechnique de Montréal, and HEC Montréal.

Expenditures as reported in Canadian dollars: (16a) $\$ 339,096$; (16b) $\$ 2,203,578$; (16c) \$0; (16d) \$0; (16) \$2,542,674; (17) \$11,481; (18a) \$721,440; (18b) \$1,087,015; (18c) \$0; (18) \$1,808,455; (20) \$50,818; (21) \$4,413,428; (22) \$55,678; (23) \$1,965,912; (24a) \$0; (24b) $\$ 0$; (25) \$0; (26) \$0. 
MONTREAL, cont.

28 In 2007-2008, 6 staffed library service points were compiled in Main branch. However, these belonged to Health branch.

\section{NEW MEXICO}

1.a Volumes held June 30, 2008 revised to 154,250. Corrected from 2008 survey submission.

5.b.ii Change from 2007-2008 figure was a result of removing titles that were freely accessible on the Internet.

18 Health Sciences Library has reorganized to provide IT support for the entire medical campus. They manage the entire staff (and salaries) associated with this support. The salaries for those staff and student employees not directly supporting the Library are no longer included in this report.

18.a-18.b Health Sciences Library has reorganized to provide IT support for the entire medical campus. They manage the entire staff (and salaries) associated with this support. The salaries for those staff not directly supporting the Library are no longer included in this report.

18.c Health Sciences Library has reorganized to provide IT support for the entire medical campus. They manage the entire staff (and salaries) associated with this support. The salaries for those students not directly supporting the Library are no longer included in this report.

20 Health Sciences Library has reorganized to provide IT support for the entire medical campus. They manage all aspects associated with this support. The other operational expenditures not directly supporting the Library are no longer included in this report.

25 Health Sciences Library has reorganized to provide IT support for the entire medical campus. They manage all aspects associated with this support. The computer hardware and software expenditures not directly supporting the Library are no longer included in this report.

27 Health Sciences Library has reorganized to provide IT support for the entire medical campus. They manage all aspects associated with this support. The FTE for staff and students not directly related to supporting the Library is no longer included in this report.

\section{NEW YORK}

All figures are as of 08/31/2009.

1.a Volumes held revised to 223,914 .

7, 9, 13 Decrease due to clean up resulting from ILS migration.

16.a Increase due to the inclusion of e-book expenditures, which were not included in the 2007-2008 figure.

16.c In prior years Ovid was counted in the Serials expenditures statistic, but it has been recategorized as "other library materials," which resulted in the increase.

37-43 Note: Overall PhD degrees/faculty/enrollment statistics provided by the NYU Office of Institutional Research and Program Evaluation; Statistics are reported in the Division of Libraries Survey.

\section{NORTHWESTERN}

All figures are as of 08/31/2009.

1.a Volumes held June 30, 2008 revised to 289,427.

\section{OKLAHOMA}

5 Serial statistics are reported as deduped for all libraries following ARL best practices.

\section{OKLAHOMA STATE}

1.a Volumes held June 30, 2008 revised to 59,441.

12 Count now based on sets of slides and sets of radiographic films rather than the previously inaccurate count of individual items.

18-18.c Includes fringe benefits.

40 This represents the number of first professional students as Medical School students are not reported in any of the IPEDS categories stated in the ARL survey.

\section{PENNSYLVANIA STATE}

Library branches included: Milton S. Hershey Medical Center College of Medicine Harrell Library Hershey, PA. 


\section{PENNSYLVANIA STATE, cont.}

\section{QUEEN'S}

$5,7, \quad$ Included in Main Library statistics.

9-10, 22,

24.a-24.b,

25-26

$16-26$

Expenditures as reported in Canadian dollars: (16a) \$127,475; (16b) $\$ 1,611,592 ;(16 c) \$ 11,309 ;$ (16d) $\$ 0 ;(16) \$ 1,750,376 ;(17) \$ 3,919$; (18a) \$653,116; (18b) \$483,549; (18c) \$36,328; (18) \$1,172,993; (20) \$190,879; (21) \$3,118,167; (22) \$0; (23) \$1,539,368; (24a) \$0; (24b) \$0; (25) $\$ 0$; (26) $\$ 0$.

\section{ROCHESTER}

Library branches included: Basil G. Bibby Library (Eastman Dental Center).

1 Does not include e-books; Rush Rhees Library reports e-books for all University of Rochester libraries.

3 Does not include e-books. HSL e-books are paid as subscriptions and are counted as serials.

$5 \quad$ For this figure, we reported print only serials plus databases. Rush Rhees Library reported all serial titles. The figure for 2007-2008 included sum of print-only serials (139) and the e-journals included in the Health Sciences and Life Sciences Categories of SFX.

9 In previous years, databases were included here. This year, we moved databases to the serials category, as per ARL instructions.

16.a Figure reflects total number of print only monographs.

16.b Figure includes serials, databases, e-book subscriptions, and instructional software.

37-43 Included in Rush Rhees Library statistics.

\section{SASKATCHEWAN}

All figures are as of 04/30/2009.

Library branches NOT included: Murray, Education, Engineering, Law, Natural Sciences, and Veterinary Medicine Libraries.

16-26 Expenditures as reported in Canadian dollars: (16a) $\$ 762,571 ;(16 b) \$ 2,361,645 ;(16 \mathrm{c}) \$ 0 ;(16 \mathrm{~d}) \$ 0 ;(16) \$ 3,124,216 ;(17) \$ 5,478$; (18a) $\$ 553,869$; (18b) $\$ 257,889$; (18c) $\$ 60,513$; (18) $\$ 872,271$; (20) $\$ 0 ;(21) \$ 4,001,965 ;(22) \$ 0 ;(23) \$ 0 ;(24 a) \$ 0 ;(24 b) \$ 0 ;(25) \$ 0 ;(26) \$ 0$.

\section{SOUTHERN CALIFORNIA}

5.a.ii Decrease due to electronic formats replacing print versions of same titles.

16.d Figure includes electronic research databases and instructional software expenditures. Increase includes multi-year contract and expense for one database.

17 Substantial reduction in print journals resulted in decrease in binding costs.

39 Health Sciences campus identifies 1,480 full-time instructional faculty. 


\section{SUNY-STONY BROOK}

1.a Volumes held June 30, 2008 revised to 273,626.

\section{TEMPLE}

5 The freely accessible titles were included in the current electronic purchased totals for 2007-2008 totals. For 2008-2009, the freely accessible titles have been separated and are included in Q5b.ii.

20 Increase is due to purchases and installation of equipment (computers, video hardware) to outfit the New Ginsburg Health Sciences Library which opened June 2009.

\section{TENNESSEE}

Library branches included: Health Sciences Library-Memphis and Preston Medical Library-Knoxville.

1.a Volumes held June 30, 2008 revised to 220,723.

5 Before deduplication, figure for total medical titles purchased was 5,263, which included 5,004 electronic titles and 259 print titles. Figure for total medical titles not purchased was 5,192.

9, 13-14 Decrease due to a more accurate item count at Health Sciences Library-Memphis.

18.c Number of student assistants increased at Preston Medical Library from 3 in 2007-2008 to 5 in 2008-2009.

29 Health Sciences Library-Memphis is open 98 hours and Preston Medical Library is open 74.5 hours per week, but these occur within the 148 hours of operation for the University Libraries.

33 Health Sciences Library-Memphis reported this item for 2008-2009 but not 2007-2008.

\section{TEXAS TECH}

All figures are as of 08/31/2009.

Library branches included: Harrington Library TTUHSC-Amarillo, Montes-Gallo Library of the Health Sciences TTUHSCEl Paso, Library of the Health Sciences TTUHSC-Odessa, Preston Smith HSC Library Lubbock.

\section{VIRGINIA}

Library branches NOT included: Alderman (Main), Astronomy, Biology/Psychology, Chemistry, Clemons Undergraduate, Education, Fiske Kimball Fine Arts, Math, Music, Physics, Brown Science \& Engineering, Small Special Collections, Law, and Darden Graduate Business.

1 Includes 2,492 e-books.

2 This figure is reported in the University Library survey for the entire University of Virginia library system.

3 Health Sciences purchased 1,639 e-books.

37-43 This figure is reported in the University Library survey for the entire University of Virginia library system.

\section{WASHINGTON}

1.a Volumes held June 30, 2008 revised to 339,036. 2007-2008 figures revised to show 36,490 volumes transferred from Health Sciences to Main.

1.b.ii $\quad 36,490$ volumes transferred from Health Sciences to Main.

5, 24, Included with Main statistics.

$37-43$

\section{WASHINGTON U.-ST. LOUIS}

1 Includes 3,963 e-books.

1.a Volumes held June 30, 2008 revised to 299,536.

18 Includes fringe benefits of $\$ 600,957$. 
Library branches NOT included: Main (GLS) (General Library System): Archives, Art, Astronomy, Biology, Business, Chemistry, College, Geography, Geology \& Geophysics, Math, Memorial, Education (Merit, formerly CIMC), Music, Physics, Primate, School of Library \& Information Science (SLIS), Social Science, Social Work, Special Collections, Steenbock (Agriculture \& Life Sciences), and Wendt (English). Non-GLS Libraries: Law, Map, Education (Merit), Primate, and Wisconsin Historical Society (American history). Also: American Indian Studies Program, Arboretum, Cooperative Children's Book Center, Center for Demography \& Ecology, Chicano \& Latino Studies, Journalism Reading Room, Max Kade German-American Institute, LGBT Campus Center, Limnology Reading Room, Learning Support Services, Morgridge Center Library, Plant Pathology Library, Space Library, Trout Lake Collection, University Communications Library, and the Wisconsin's Water Library.

5 Total serial title holdings, in all formats, cannot be provided for one specific campus library. The total number of serial titles held for University of Wisconsin (UW) Madison is 101,596. This number represents all reporting UW Madison campus libraries, including Health Sciences (Ebling) Library.

18-18.c Includes fringe benefits.

30-31 Reduction from 2007-2008 figure is based upon current review of campus reporting for small-group instruction and consultations.

37-43 Numbers reported for entire University of Wisconsin-Madison campus, regardless of discipline affiliation, school or college.

YALE

1.a Volumes held June 30, 2008 revised to 462,238. 


\section{ARL Member Libraries as of January 1, 2010}

The Association of Research Libraries (ARL) represents the interests of 124 libraries that serve major North American research institutions. The ARL Statistics and Measurement program is organized around identifying, collecting, analyzing, and distributing quantifiable information describing the characteristics of research libraries.

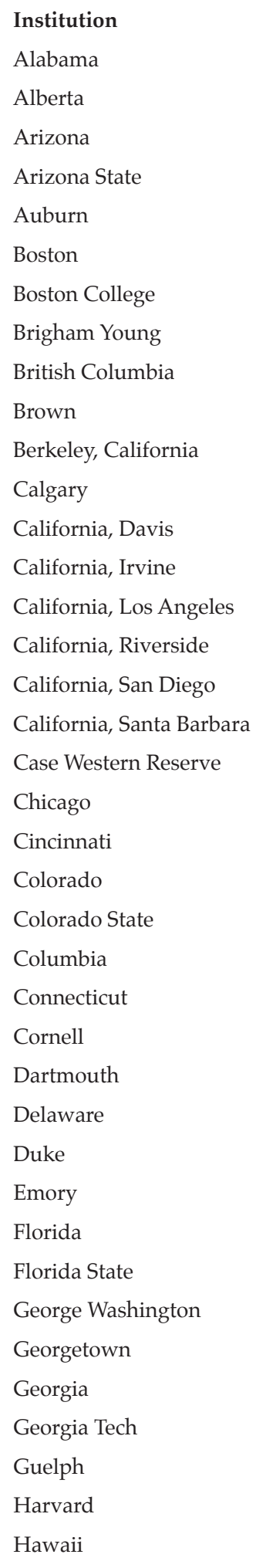

\begin{tabular}{|c|c|}
\hline Category & Full Name of Institution \\
\hline$S$ & University of Alabama \\
\hline $\mathrm{C}$ & University of Alberta \\
\hline S & University of Arizona \\
\hline$S$ & Arizona State University \\
\hline$S$ & Auburn University \\
\hline $\mathrm{P}$ & Boston University \\
\hline $\mathrm{P}$ & Boston College \\
\hline $\mathrm{P}$ & Brigham Young University \\
\hline $\mathrm{C}$ & University of British Columbia \\
\hline $\mathrm{P}$ & Brown University \\
\hline$S$ & University of California, Berkeley \\
\hline $\mathrm{C}$ & University of Calgary \\
\hline S & University of California, Davis \\
\hline S & University of California, Irvine \\
\hline S & University of California, Los Angeles \\
\hline S & University of California, Riverside \\
\hline S & University of California, San Diego \\
\hline S & University of California, Santa Barbara \\
\hline $\mathrm{P}$ & Case Western Reserve University \\
\hline $\mathrm{P}$ & University of Chicago \\
\hline S & University of Cincinnati \\
\hline S & University of Colorado \\
\hline S & Colorado State University \\
\hline $\mathrm{P}$ & Columbia University \\
\hline S & University of Connecticut \\
\hline $\mathrm{P}$ & Cornell University \\
\hline $\mathrm{P}$ & Dartmouth College \\
\hline S & University of Delaware \\
\hline $\mathrm{P}$ & Duke University \\
\hline $\mathrm{P}$ & Emory University \\
\hline S & University of Florida \\
\hline S & Florida State University \\
\hline $\mathrm{P}$ & George Washington University \\
\hline $\mathrm{P}$ & Georgetown University \\
\hline S & University of Georgia \\
\hline S & Georgia Institute of Technology \\
\hline $\mathrm{C}$ & University of Guelph \\
\hline $\mathrm{P}$ & Harvard University \\
\hline S & University of Hawaii \\
\hline
\end{tabular}

\section{Location}

Tuscaloosa, Alabama

Edmonton, Alberta

Tucson, Arizona

Tempe, Arizona

Auburn, Alabama

Boston, Massachusetts

Boston, Massachusetts

Provo, Utah

Vancouver, British Columbia

Providence, Rhode Island

California, Berkeley

Calgary, Alberta

Davis, California

Irvine, California

Los Angeles, California

Riverside, California

La Jolla, California

Santa Barbara, California

Cleveland, Ohio

Chicago, Illinois

Cincinnati, Ohio

Boulder, Colorado

Fort Collins, Colorado

New York, New York

Storrs, Connecticut

Ithaca, New York

Hanover, New Hampshire

Newark, Delaware

Durham, North Carolina

Atlanta, Georgia

Gainesville, Florida

Tallahassee, Florida

Washington, DC

Washington, DC

Athens, Georgia

Atlanta, Georgia

Guelph, Ontario

Cambridge, Massachusetts

Honolulu, Hawaii 


\begin{tabular}{|c|c|c|}
\hline Institution & Category & Full Name of Institution \\
\hline Houston & S & University of Houston \\
\hline Howard & $\mathrm{P}$ & Howard University \\
\hline Illinois, Chicago & $S$ & University of Illinois at Chicago \\
\hline Illinois, Urbana & S & University of Illinois at Urbana \\
\hline Indiana & $S$ & Indiana University \\
\hline Iowa & $\mathrm{S}$ & University of Iowa \\
\hline Iowa State & S & Iowa State University \\
\hline Johns Hopkins & $\mathrm{P}$ & Johns Hopkins University \\
\hline Kansas & S & University of Kansas \\
\hline Kent State & $S$ & Kent State University \\
\hline Kentucky & $S$ & University of Kentucky \\
\hline Laval & $\mathrm{C}$ & Laval University \\
\hline Louisiana State & $S$ & Louisiana State University \\
\hline Louisville & $S$ & University of Louisville \\
\hline McGill & $\mathrm{C}$ & McGill University \\
\hline McMaster & $\mathrm{C}$ & McMaster University \\
\hline Manitoba & $\mathrm{C}$ & University of Manitoba \\
\hline Maryland & $S$ & University of Maryland \\
\hline Massachusetts & S & University of Massachusetts \\
\hline MIT & $\mathrm{P}$ & Massachusetts Institute of Technology \\
\hline Miami & $\mathrm{P}$ & University of Miami \\
\hline Michigan & S & University of Michigan \\
\hline Michigan State & $S$ & Michigan State University \\
\hline Minnesota & $S$ & University of Minnesota \\
\hline Missouri & S & University of Missouri \\
\hline Montreal & $\mathrm{C}$ & University of Montreal \\
\hline Nebraska & $S$ & University of Nebraska-Lincoln \\
\hline New Mexico & S & University of New Mexico \\
\hline New York & $\mathrm{P}$ & New York University \\
\hline North Carolina & $S$ & University of North Carolina \\
\hline North Carolina State & $S$ & North Carolina State University \\
\hline Northwestern & $\mathrm{P}$ & Northwestern University \\
\hline Notre Dame & $\mathrm{P}$ & University of Notre Dame \\
\hline Ohio & $S$ & Ohio University \\
\hline Ohio State & S & Ohio State University \\
\hline Oklahoma & $S$ & University of Oklahoma \\
\hline Oklahoma State & $S$ & Oklahoma State University \\
\hline Oregon & $S$ & University of Oregon \\
\hline Pennsylvania & $\mathrm{P}$ & University of Pennsylvania \\
\hline Pennsylvania State & $S$ & Pennsylvania State University \\
\hline Pittsburgh & $S$ & University of Pittsburgh \\
\hline Princeton & $\mathrm{P}$ & Princeton University \\
\hline Purdue & S & Purdue University \\
\hline Queen's & $\mathrm{C}$ & Queen's University \\
\hline
\end{tabular}

\section{Location}

Houston, Texas

Washington, DC

Chicago, Illinois

Urbana, Illinois

Bloomington, Indiana

Iowa City, Iowa

Ames, Iowa

Baltimore, Maryland

Lawrence, Kansas

Kent, Ohio

Lexington, Kentucky

Quebec, Quebec

Baton Rouge, Louisiana

Louisville, Kentucky

Montreal, Quebec

Hamilton, Ontario

Winnipeg, Manitoba

College Park, Maryland

Amherst, Massachusetts

Cambridge, Massachusetts

Coral Gables, Florida

Ann Arbor, Michigan

East Lansing, Michigan

Minneapolis, Minnesota

Columbia, Missouri

Montreal, Quebec

Lincoln, Nebraska

Albuquerque, New Mexico

New York, New York

Chapel Hill, North Carolina

Raleigh, North Carolina

Evanston, Illinois

Notre Dame, Indiana

Athens, Ohio

Columbus, Ohio

Norman, Oklahoma

Stillwater, Oklahoma

Eugene, Oregon

Philadelphia, Pennsylvania

University Park, Pennsylvania

Pittsburgh, Pennsylvania

Princeton, New Jersey

West Lafayette, Indiana

Kingston, Ontario 


\begin{tabular}{|c|c|c|c|}
\hline Institution & Category & Full Name of Institution & Location \\
\hline Rice & $\mathrm{P}$ & Rice University & Houston, Texas \\
\hline Rochester & $\mathrm{P}$ & University of Rochester & Rochester, New York \\
\hline Rutgers & $S$ & Rutgers University & New Brunswick, New Jersey \\
\hline Saskatchewan & $\mathrm{C}$ & University of Saskatchewan & Saskatoon, Saskatchewan \\
\hline South Carolina & S & University of South Carolina & Columbia, South Carolina \\
\hline Southern California & $\mathrm{P}$ & University of Southern California & Los Angeles, California \\
\hline Southern Illinois & $S$ & Southern Illinois University & Carbondale, Illinois \\
\hline SUNY-Albany & S & University at Albany, State University of New York & Albany, New York \\
\hline SUNY-Buffalo & S & University at Buffalo, State University of New York & Buffalo, New York \\
\hline SUNY-Stony Brook & $S$ & State University of New York at Stony Brook & Stony Brook, New York \\
\hline Syracuse & $\mathrm{P}$ & Syracuse University & Syracuse, New York \\
\hline Temple & S & Temple University & Philadelphia, Pennsylvania \\
\hline Tennessee & $S$ & University of Tennessee & Knoxville, Tennessee \\
\hline Texas & $S$ & University of Texas & Austin, Texas \\
\hline Texas A\&M & S & Texas A\&M University & College Station, Texas \\
\hline Texas Tech & S & Texas Tech University & Lubbock, Texas \\
\hline Toronto & $\mathrm{C}$ & University of Toronto & Toronto, Ontario \\
\hline Tulane & $\mathrm{P}$ & Tulane University & New Orleans, Louisiana \\
\hline Utah & S & University of Utah & Salt Lake City, Utah \\
\hline Vanderbilt & $\mathrm{P}$ & Vanderbilt University & Nashville, Tennessee \\
\hline Virginia & S & University of Virginia & Charlottesville, Virginia \\
\hline Virginia Tech & S & Virginia Polytechnic Institute \& State University & Blacksburg, Virginia \\
\hline Washington & S & University of Washington & Seattle, Washington \\
\hline Washington State & S & Washington State University & Pullman, Washington \\
\hline Washington U.-St. Louis & $\mathrm{P}$ & Washington University & St. Louis, Missouri \\
\hline Waterloo & $\mathrm{C}$ & University of Waterloo & Waterloo, Ontario \\
\hline Wayne State & S & Wayne State University & Detroit, Michigan \\
\hline Western Ontario & $\mathrm{C}$ & University of Western Ontario & London, Ontario \\
\hline Wisconsin & S & University of Wisconsin & Madison, Wisconsin \\
\hline Yale & $\mathrm{P}$ & Yale University & New Haven, Connecticut \\
\hline York & $\mathrm{C}$ & York University & North York, Ontario \\
\hline Boston Public Library & $\mathrm{N}$ & Boston Public Library & Boston, Massachusetts \\
\hline Canada Inst. SciTech Info. & $X$ & Canada Inst. for Scientific \& Technical Information & Ottawa, Ontario \\
\hline Center for Research Libraries & $\mathrm{N}$ & Center for Research Libraries & Chicago, Illinois \\
\hline Library of Congress & $\mathrm{N}$ & Library of Congress & Washington, DC \\
\hline National Agricultural Library & $\mathrm{N}$ & National Agricultural Library & Beltsville, Maryland \\
\hline Library and Archives Canada & $x$ & Library and Archives Canada & Ottawa, Ontario \\
\hline Naionat. Library of Medicine & $\mathrm{N}$ & National Library of Medicine & Bethesda, Maryland \\
\hline New York Public Library & $\mathrm{N}$ & New York Public Library & New York, New York \\
\hline New York State Library & $\mathrm{N}$ & New York State Library & Albany, New York \\
\hline Smithsonian Institution & $\mathrm{N}$ & Smithsonian Institution & Washington, DC \\
\hline $\begin{array}{l}\text { S: US public university } \\
\text { P: US private university } \\
\text { C: Canadian university } \\
\text { N: US nonuniversity } \\
\text { X: Canadian nonuniversity }\end{array}$ & & & \\
\hline
\end{tabular}


